COMMUNICATIONS IN

ANALYSIS AND GEOMETRY

Volume 14, Number 5, 907-943, 2006

\title{
Harmonic mean curvature flow on surfaces of negative Gaussian curvature
}

\author{
Panagiota Daskalopoulos and Richard Hamilton
}

\begin{abstract}
We consider the evolution of a surface of revolution with boundary $\Sigma(t)$ in $\mathbb{R}^{3}$ by the harmonic mean curvature flow (HMCF) where each point $P$ moves in the normal inward direction with velocity equal to the harmonic mean curvature of the surface. We assume that the principal eigenvalues $\lambda_{1}$ and $\lambda_{2}$ of the initial surface have opposite signs, namely $K=\lambda_{1} \lambda_{2}<0$, while $H=\lambda_{1}+\lambda_{2}<0$. We show that there exists a time $T_{0}>0$ for which the (HMCF ) admits a unique solution $\Sigma(t)$ up to $T_{0}$ such that $H<0$ for all $t<T_{0}$ and $\tilde{H}\left(\cdot, T_{0}\right) \equiv 0$ on some set of sufficiently large measure. In addition, the boundary of the surface evolves by the curve shortening flow.
\end{abstract}

\section{Introduction}

We consider the evolution of a surface $\Sigma(t)$ in $\mathbb{R}^{3}$ by the harmonic mean curvature flow (HMCF)

$$
\frac{\partial P}{\partial t}=\frac{K}{H} \vec{N}
$$

where each point $P$ moves in the inward direction $\vec{N}$ with velocity equal to the harmonic mean curvature of the surface, namely the quotient $K / H$ of the Gaussian curvature $K$ of the surface over the its mean curvature H. Denoting by $\lambda_{1}, \lambda_{2}$ the two principal curvatures of the surface, we may express the harmonic mean curvature $K / H$ of the surface as

$$
\frac{K}{H}=\frac{\lambda_{1} \lambda_{2}}{\lambda_{1}+\lambda_{2}}=\frac{1}{\lambda_{1}^{-1}+\lambda_{2}^{-1}} .
$$

The evolution of a strictly convex surface $\Sigma(t)$ under the HMCF has been studied by Andrews [1,2], where existence and convergence to a round sphere was shown. The existence for weakly convex surfaces with $H>0$ has been shown by Diater [3].

In this work, we will consider the case where the principal eigenvalues $\lambda_{1}$ and $\lambda_{2}$ have opposite signs, namely $K=\lambda_{1} \lambda_{2}<0$. We will restrict ourselves 
to surfaces of revolution $r=f(x, t)$ around the $x$-axis. For such a surface of revolution, the two principal curvatures are given by

$$
\lambda_{1}=-\frac{f_{x x}}{\left(1+f_{x}^{2}\right)^{3 / 2}} \quad \text { and } \quad \lambda_{2}=\frac{1}{f\left(1+f_{x}^{2}\right)^{1 / 2}}
$$

Hence,

$$
H=\lambda_{1}+\lambda_{2}=\frac{-f f_{x x}+f_{x}^{2}+1}{f\left(1+f_{x}^{2}\right)^{3 / 2}}
$$

and

$$
K=\lambda_{1} \lambda_{2}=\frac{-f_{x x}}{f\left(1+f_{x}^{2}\right)^{2}} .
$$

When the surface $r=f(x, t)$ evolves by the HMCF, the function $f$ evolves by the fully nonlinear equation

$$
\frac{\partial f}{\partial t}=\frac{f_{x x}}{-f f_{x x}+f_{x}^{2}+1} .
$$

We will consider solutions of (1.1) on the interval $I=[0,1]$ such that

$$
f>0 \quad \text { and } \quad f_{x x}>0 \text { on } I
$$

and

$$
\tilde{H}=-f f_{x x}+f_{x}^{2}+1<0 \quad \text { on } I .
$$

Under the above assumptions $f_{t} \leq 0$, which makes $f$ to decrease, i.e., the surface of revolution shrinks. Equation (1.1) becomes singular when the denominator $\tilde{H}$ first becomes zero, i.e., when the mean curvature $H$ of the surface becomes zero. We will show in this paper that if the initial surface satisfies conditions (1.2) and (1.3) and the boundary growth condition

$$
c \leq x^{2-p}(1-x)^{2-p} f_{x x} \leq C, \quad 0<x<1
$$

for some number $0<p<1$ and some constants $c>0$ and $C<\infty$, then there exists a time $T_{0}>0$ where first $\tilde{H}=0$ while conditions (1.2) and (1.4) hold on $0 \leq t \leq T_{0}$. In addition, we will show that there exists a constant $l_{0}>0$, depending on the initial surface, such that $\tilde{H}\left(\cdot, T_{0}\right) \equiv 0$ on some interval $I_{0}$ of length $\left|I_{0}\right| \geq l_{0}$. 
Under the growth assumptions (1.4), the equation becomes degenerate at the boundary points $x_{i}=0,1$. As a consequence, $f\left(x_{i}, t\right)$ satisfy

$$
f_{t}\left(x_{i}, t\right)=-\frac{1}{f\left(x_{i}, t\right)}, \quad \text { at } x_{i}=0,1
$$

i.e., the boundary of the surface of revolution $z=f(r, t)$ moves by the curve shortening flow.

To understand better the behavior of Equation (1.1) let us linearize the equation around a point $f$. We obtain the equation

$$
\tilde{f}_{t}=\frac{1+f_{x}^{2}}{\tilde{H}^{2}} \tilde{f}_{x x}-\frac{2 f_{x} f_{x x}}{\tilde{H}^{2}} \tilde{f}_{x}+\frac{f_{x x}^{2}}{\tilde{H}^{2}} \tilde{f}
$$

with $\tilde{H}=-f f_{x x}+f_{x}^{2}+1$.

We observe that the principal coefficient of the linearized equation is non-negative independently of the sign of $\tilde{H}$ and $f_{x x}$, i.e., independently of the sign of the mean curvature $H$ and the Gaussian curvature $K$ of the surface of revolution. This property distinguishes the HMCF from other previously studied geometric flows, such as the Gauss curvature flow, which becomes backwards parabolic at points of negative Gaussian curvature.

Also, if $f$ satisfies conditions (1.2)-(1.4), all the coefficients of Equation (1.6) are bounded on $I$ and the principal coefficient is bounded away from zero in the interior of $I$. However, the equation becomes degenerate at the boundary points $x_{i}=0,1$ and takes the degenerate form

$$
\tilde{f}_{t}=\left(x-x_{i}\right)^{2} a(x, t) \tilde{f}_{x x}+\left(x-x_{i}\right) b(x, t) \tilde{f}_{x}+c(x, t) \tilde{f},
$$

where all coefficients $a, b, c$ are bounded on $I$ and $a$ is bounded away from zero on $I$. Hence, near the boundary points $x_{i}=0,1$, the evolution is governed by the hyperbolic metric $d s_{i}^{2}=d x^{2} /\left(x-x_{i}\right)^{2}$.

For $0<\alpha<1$, let us denote by $C_{s}^{\alpha}(I)$ the set of all Hölder continuous functions on $I$ with respect to a fixed metric $d s^{2}$ which is equivalent to $d s_{i}^{2}=d x^{2} /\left(x-x_{i}\right)^{2}$ near the boundary points $x_{i}=0,1$ while it is equivalent to the standard metric in the interior of $I$.

Also, for $0<p<1$, let us define $C_{s}^{2+\alpha, p}(I)$ to be the space of all functions $g$ on $I$ such that

$$
\left|x-x_{i}\right|^{-p}\left(g-g\left(x_{i}\right)\right), \quad\left|x-x_{i}\right|^{1-p} g_{x}, \quad\left|x-x_{i}\right|^{2-p} g_{x x} \in C_{s}^{\alpha}
$$


with $x_{i}=0,1$. Also, for $T>0$, we set $Q_{T}=I \times[0, T]$ and we define $C_{s}^{2+\alpha, p}\left(Q_{T}\right)$ to be the space of all functions $g$ on $Q_{T}$ such that $\tilde{g}^{i}(x, t)=$ $g(x, t)-g\left(x_{i}, t\right), x_{i}=0,1$ satisfies

$$
\begin{gathered}
\left|x-x_{i}\right|^{-p} \tilde{g}^{i}, \quad\left|x-x_{i}\right|^{-p} \tilde{g}_{t}^{i}, \quad\left|x-x_{i}\right|^{1-p} \tilde{g}_{x}^{i}, \\
\left|x-x_{i}\right|^{2-p} \tilde{g}_{x x}^{i} \in C_{s}^{\alpha}\left(Q_{T}\right)
\end{gathered}
$$

where $C_{s}^{\alpha}\left(Q_{T}\right)$ denotes the space of Hölder continuous functions on $Q_{T}$ with respect to the parabolic distance $\bar{d} s^{2}=d s^{2}+|d t|$. Finally, for $k \geq 2$, $0<p<1$, we define $C^{k, p}\left(Q_{T}\right)$ to be the space of all functions $g \in C^{0}\left(Q_{T}\right)$ on $Q_{T}$ such that $\tilde{g}^{i}(x, t)=g(x, t)-g\left(x_{i}, t\right), x_{i}=0,1$, satisfies

$$
x^{j-p}(1-x)^{j-p}\left|D_{t}^{l} D_{x}^{j} \tilde{g}^{i}\right| \leq C, \quad 1 \leq 2 l+j \leq k
$$

on $Q_{T}$, and we set

$$
C^{\infty, p}\left(Q_{T}\right)=\cap_{k} C^{k, p}\left(Q_{T}\right) .
$$

The main result in this paper states as follows:

Theorem 1.1. Assume that at time $t=0, \Sigma$ is a surface of revolution $r=$ $f(x, 0)$, with $f(\cdot, 0) \in C_{s}^{2+\alpha, p}(I)$ for some $0<\alpha, p<1$ and satisfies conditions (1.2)-(1.4). Then, there exists a time $T_{0}>0$ for which the HMCF (1.1) admits a unique solution $f \in C^{2, p}\left(Q_{T_{0}}\right)$ on $Q_{T_{0}}=I \times\left[0, T_{0}\right]$ such that $\tilde{H}=$ $-f f_{x x}+f_{x}^{2}+1<0$ for all $t<T_{0}$ and $\tilde{H}\left(\cdot, T_{0}\right) \equiv 0$ on some interval $I_{0} \subset$ $[0,1]$ of length

$$
\left|I_{0}\right| \geq l_{0}
$$

with $l_{0}>0$ depending on the initial data. In addition, $f \in C^{\infty, p}\left(Q_{T}\right)$ for all $T<T_{0}$, and it satisfies the boundary condition (1.5).

In Section 2, we will establish the short-time existence of the HMCF for a surface of revolution under the initial assumptions of Theorem 1.1. In Section 3, we will establish the short-time $C^{\infty, p}$ regularity of the solution. We will also show that the area of the surface remains constant under the flow. In Section 5, we will show that the flow exists and it is smooth up to the time $T_{0}$ where $\tilde{H}$ first reaches zero. This result will be based on sharp a priori derivative bounds up to $T_{0}$, which will be established in Section 4 . Finally, in Section 6 , we will show that $\tilde{H}\left(\cdot, T_{0}\right) \equiv 0$ on an interval $I_{0} \subset I$ of sufficiently large length. 


\section{Short-time existence and uniqueness}

In this section, we will establish the short-time existence for Equation (1.1) under the initial assumptions of Theorem 1.1 as stated in the following result.

Theorem 2.1. Assume that at time $t=0, \Sigma$ is a surface of revolution $r=$ $f(x, 0)$, with $f(\cdot, 0) \in C_{s}^{2+\alpha, p}(I)$ for some $0<\alpha, p<1$. In addition, assume that $f(\cdot, 0)$ satisfies conditions (1.2)-(1.4). Then, there exists $\tau_{0}>0$ for which the HMCF (1.1) admits a unique solution $f \in C_{s}^{2+\alpha, p}\left(Q_{\tau_{0}}\right)$ on $Q_{\tau_{0}}=$ $I \times\left[0, \tau_{0}\right]$, which satisfies conditions (1.2)-(1.5).

The short-time existence for the highly degenerate HMCF on weakly convex surfaces with flat sides was recently shown by Caputo and Daskalopoulos [4], without the assumption of radial symmetry. Since the equation becomes degenerate on the flat side, similar boundary conditions to (1.2)(1.4) need to be imposed near the flat side. The boundary of the flat side evolves by the curve shortening flow as well.

To simplify the computations and the notation, we will assume that $f(0, t)=f(1, t)$, at $t=0$, which will imply that

$$
f(0, t)=f(1, t)=d(t), \quad \forall t<T_{0}
$$

once we show that $f\left(x_{i}, t\right), x_{i}=0,1$ evolve by (1.5). This assumption is only technical and can be easily removed.

Local change of coordinates. Since we expect the linearized Equation (1.6) to be of the form (1.7), we introduce near the boundary point $x=0$ the change of variables

$$
h(z, t)=f(x, t)-f(0, t), \quad z=\log x .
$$

Then $z \rightarrow-\infty$, as $x \rightarrow 0$. Denoting by $d(t)=f(0, t)$ and using the equation $d^{\prime}(t)=-1 / d(t)$ (as implied by (1.5)) we find by direct computation that $h$ satisfies the equation

$$
h_{t}=\frac{h_{z z}-h_{z}}{\mathrm{e}^{2 z}+h_{z}^{2}-(h+d(t))\left(h_{z z}-h_{z}\right)}+\frac{1}{d(t)} .
$$

The linearized equation around a point $h$ is

$$
\tilde{h}_{t}=\alpha(h, z) \tilde{h}_{z z}+\beta(h, z) \tilde{h}_{z}+\gamma(h, z) \tilde{h}
$$


with

$$
\begin{aligned}
& \alpha(h, z)=\frac{\mathrm{e}^{2 z}+h_{z}^{2}}{\left(\mathrm{e}^{2 z}+h_{z}^{2}-(h+d(t))\left(h_{z z}-h_{z}\right)\right)^{2}} \\
& \beta(h, z)=\frac{-\mathrm{e}^{2 z}+h_{z}^{2}-2 h_{z} h_{z z}}{\left(\mathrm{e}^{2 z}+h_{z}^{2}-(h+d(t))\left(h_{z z}-h_{z}\right)\right)^{2}} \\
& \gamma(h, z)=\frac{\left(h_{z z}-h_{z}\right)^{2}}{\left(\mathrm{e}^{2 z}+h_{z}^{2}-(h+d(t))\left(h_{z z}-h_{z}\right)\right)^{2}} .
\end{aligned}
$$

We will show that under the assumptions of Theorem 2.1, the linearized Equation (2.2) is non-degenerate.

Similarly, near the boundary point $x=1$, we introduce the change of variables

$$
h(z, t)=f(x, t)-f(1, t), \quad z=-\log (1-x)
$$

so that this time $z \rightarrow+\infty$, as $x \rightarrow 1$. The function $h$ satisfies this time the equation

$$
h_{t}=\frac{h_{z z}-h_{z}}{\mathrm{e}^{-2 z}+h_{z}^{2}-(h+d(t))\left(h_{z z}-h_{z}\right)}+\frac{1}{d(t)}
$$

and the linearized equation is non-degenerate and similar to (2.2).

For $T>0$, we set $S_{T}=\mathbb{R} \times[0, T]$ and define the space $C^{2+\alpha, p}\left(S_{T}\right)$ of all functions $h$ on $S_{T}$ such that $\mathrm{e}^{p z} h \in C^{2+\alpha}\left(S_{T}\right)$ and $\mathrm{e}^{-p z} h \in C^{2+\alpha}\left(S_{T}\right)$ with norm

$$
\|h\|_{C^{2+\alpha, p}\left(S_{T}\right)}=\left\|\mathrm{e}^{p z} h\right\|_{C^{2+\alpha}\left(S_{T}\right)}+\left\|\mathrm{e}^{-p z} h\right\|_{C^{2+\alpha}\left(S_{T}\right)} .
$$

Similarly, we denote by $C^{\alpha, p}\left(S_{T}\right)$ the space of all functions $h$ on $S_{T}$ such that $\mathrm{e}^{p z} h \in C^{\alpha}\left(S_{T}\right)$ and $\mathrm{e}^{-p z} h \in C^{\alpha}\left(S_{T}\right)$ with norm

$$
\|h\|_{C^{\alpha, p}\left(S_{T}\right)}=\left\|\mathrm{e}^{p z} h\right\|_{C^{\alpha}\left(S_{T}\right)}+\left\|\mathrm{e}^{-p z} h\right\|_{C^{\alpha}\left(S_{T}\right)} .
$$

Here, $C^{2+\alpha}\left(S_{T}\right)$ denotes the standard space of all functions $g$ such that

$$
g, g_{t}, g_{z}, g_{z z} \in C^{\alpha}\left(S_{T}\right)
$$

with respect to the standard parabolic distance.

Global change of coordinates. To prove Theorem 2.1, we need to introduce a global coordinate change which near the boundary points $x_{i}=0,1$ is equal to the local coordinate change we introduced above. Let $\theta$ be a smooth, 
non-increasing function on $[0,1]$ such that $\theta=1$ on $0 \leq x \leq \mathrm{e}^{-1}$ and $\theta=0$ on $1-\mathrm{e}^{-1} \leq x \leq 1$. We set

$$
h(z, t)=f(x, t)-d(t), \quad z=\theta(x) \log x-(1-\theta(x)) \log (1-x) .
$$

It is clear that $z=\log x, x \in\left[0, \mathrm{e}^{-1}\right]$ and $z=-\log (1-x), x \in\left[1-\mathrm{e}^{-1}, 1\right]$. Hence, the function $h$ satisfies Equation $(2.1)$ on $z \in(-\infty,-1]$ and Equation (2.6) on $z \in[1,+\infty)$. On the interval $[-1,1]$, $h$ satisfies a strictly parabolic fully nonlinear equation as long as $\tilde{H}>0$.

The condition that $f \in C_{s}^{2+\alpha, p}\left(Q_{T}\right)$ is equivalent to the condition that $h \in C^{2+\alpha, p}\left(S_{T}\right)$. In terms of the new coordinates, conditions (1.2) and (1.3) may be expressed

$$
h+d(t)>0 \quad \text { and } \quad h_{z z}-h_{z}>0 \quad \text { on } \mathbb{R}
$$

and

$$
\tilde{H}=-(h+d(t)) h_{z z}+h_{z}^{2}+\mathrm{e}^{2 z}<0 \quad \text { on } \mathbb{R}
$$

and while assuming that $h \in C^{2+\alpha, p}\left(S_{T}\right)$, condition (1.4) is equivalent to the non-degeneracy condition

$$
\left(\mathrm{e}^{-p z}+\mathrm{e}^{p z}\right)\left(h_{z z}-h_{z}\right) \geq \mu, \quad \forall z \in \mathbb{R}
$$

for some number $\mu>0$.

It follows that the linearized equation around a function $h \in C^{2+\alpha, p}\left(S_{T}\right)$ which satisfies conditions (2.8)-(2.10), is of the form

$$
w_{t}=\tilde{\alpha}(z, t) w_{z z}+\tilde{\beta}(z, t) w_{z}+\tilde{\gamma}(z, t) w
$$

with

$$
\inf _{S_{T}} \tilde{\alpha}(z, t) \geq \lambda
$$

and

$$
\|\tilde{\alpha}\|_{C^{\alpha}\left(S_{T}\right)}+\|\beta\|_{C^{\alpha}\left(S_{T}\right)}+\|\tilde{\gamma}\|_{C^{\alpha}\left(S_{T}\right)} \leq \lambda^{-1}
$$

for some number $0<\lambda<\infty$. Let us denote by $L$ the operator

$$
L w=w_{t}-\left[\tilde{\alpha}(z, t) w_{z z}+\tilde{\beta}(z, t) w_{z}+\tilde{\gamma}(z, t) w\right] .
$$


The following result is a consequence of the classical Schauder estimates for linear parabolic equations.

Proposition 2.2. Assume that $w_{0} \in C^{2+\alpha, p}(\mathbb{R})$ and $g \in C^{2+\alpha, p}\left(S_{T}\right)$ for some numbers $0<\alpha, p<1$. Then, there exists a unique solution $w \in$ $C^{2+\alpha, p}\left(S_{T}\right)$ of the initial value problem $L w=g$ in $S_{T}, w(\cdot, 0)=w_{0}$, satisfying

$$
\|w\|_{C^{2+\alpha, p}\left(S_{T}\right)} \leq C(T)\left(\left\|w_{0}\right\|_{C^{2+\alpha, p}(\mathbb{R})}+\|g\|_{C^{\alpha, p}\left(S_{T}\right)}\right)
$$

for any $T>0$. The constant $C(T)$ depends only on the numbers $\alpha, p$ and $\lambda$.

Lemma 2.3. Assume that at time $t=0, h(\cdot, 0) \in C^{2+\alpha, p}(\mathbb{R}), 0<\alpha, p<1$. In addition, assume that $h_{0}=h(\cdot, 0)$ satisfies conditions $(2.8)-(2.10)$. Then, there exists $\tau_{0}>0$ for which the HMCF admits a solution $h \in C^{2+\alpha, p}\left(S_{\tau_{0}}\right)$ on $S_{\tau_{0}}=\mathbb{R} \times\left[0, \tau_{0}\right]$.

Proof. For $\tau>0$, let us denote by $M: C^{2+\alpha, p}\left(S_{\tau}\right) \rightarrow C^{\alpha, p}\left(S_{\tau}\right)$ the fully nonlinear operator

$$
M(h)=h_{t}-F\left(z, t, h, h_{z}, h_{z z}\right)
$$

with

$$
F\left(z, t, h, h_{z}, h_{z z}\right)=\frac{h_{z z}-h_{z}}{\mathrm{e}^{2 z}+h_{z}^{2}-(h+d(t))\left(h_{z z}-h_{z}\right)}+\frac{1}{d(t)} .
$$

It is easy to observe that the linearization

$$
w_{t}=D F(\tilde{h})(w)
$$

around a point $\tilde{h} \in C^{2+\alpha, p}\left(S_{\tau}\right)$ satisfies all the hypotheses of Proposition 2.2, provided that $\left\|\tilde{h}-h_{0}\right\|_{C^{2+\alpha, p}\left(S_{\tau}\right)}<\delta$, with $\delta$ sufficiently small, depending on the initial data.

Hence, combining Proposition 2.2 and the inverse function theorem between Banach spaces, we conclude that there exists a number $\tau_{0}>0$ depending on the initial data, for which the initial value problem

$$
\left\{\begin{array}{l}
h_{t}=F\left(z, t, h, h_{z}, h_{z z}\right) \\
h(z, 0)=h_{0}
\end{array}\right.
$$

admits a solution $h$ in the space $C^{2+\alpha, p}\left(S_{\tau_{0}}\right)$, finishing the proof of the lemma. For more details, we refer the reader to the proof of Theorem 8.5 in $[5]$. 
Proof of Theorem 2.1. The existence of the solution $f$ of (1.1) on $Q_{\tau_{0}}$ satisfying conditions (1.2)-(1.4) follows directly from Lemma 2.3 by expressing $h$ back to the original coordinates.

To see that $f$ also satisfies (1.5), one simply takes the limit $x \rightarrow x_{i}$ on Equation (1.1), observing that by condition (1.4) (with $0<p<1$ ) both $f_{x x}$ and $f_{x}^{2}$ tend to infinity as $x \rightarrow x_{i}$, while $f_{x x} / f_{x}^{2} \rightarrow 0$.

The uniqueness of such a solution $f$ follows by observing that any two solutions $f_{1}, f_{2}$ of (1.1) with the same initial data will coincide at the boundary points $x_{i}=0,1$ for all times $0 \leq t \leq \tau_{0}$, as they satisfy (1.5). Hence, the classical comparison principle applies in this case.

\section{Higher regularity and area formula}

We will establish next the higher regularity of a solution $f \in C^{2+\alpha, p}\left(Q_{T}\right)$ of (1.1) up to the boundary, which will be used in the proof of Theorem 1.1.

For $0<\alpha, p<1$ and $k \geq 2$, we define $C_{s}^{k+\alpha, p}\left(Q_{T}\right)$ to be the space of all functions $g$ on $Q_{T}$ such that

$$
g,\left|x-x_{i}\right|^{j-p} D_{t}^{l} D_{x}^{j} g \in C_{s}^{\alpha}\left(Q_{T}\right), \quad x_{i}=0,1, \quad 1 \leq 2 l+j \leq k,
$$

where $C_{s}^{\alpha}\left(Q_{T}\right)$ denotes the space of Hölder continuous functions on $Q_{T}$ with respect to the hyperbolic-parabolic distance $\bar{d} s^{2}=d s^{2}+|d t|$ (as defined in the Introduction).

Proposition 3.1. Assume that $f \in C_{s}^{2+\alpha, p}\left(Q_{T}\right)$ is a solution of the $H M C F$ (1.1) which satisfies conditions (1.2)-(1.4) on $Q_{T}$. Then, $f \in C_{s}^{k+\alpha, p}\left(Q_{T}\right)$, for any $k \geq 2$. Hence, $f \in C^{\infty, p}\left(Q_{T}\right)$.

Proof. The $C^{\infty}$ regularity of $f$ in the interior of the interval $I$ readily follows by classical regularity theory and conditions (1.2)-(1.4). Hence, we only need to show the regularity of $f$ near the boundary points $x_{i}=0,1$.

To show the regularity near $x=0$, we set

$$
h(z, t)=f(x, t)-d(t), \quad z=\log x, d(t)=f(0, t)
$$

and compute, as in the previous section, that $h$ satisfies (2.1). Since $\tilde{h}:=h_{z}$ satisfies (2.2) with coefficients given, in terms of $h$, by (2.3)-(2.5), and $h \in$ $C^{2+\alpha}((-\infty,-1] \times[0, T])$, we conclude that the coefficients of $(2.2)$ satisfy conditions (2.12) and (2.13). Hence, the regularity $\mathrm{e}^{-p z} h_{z} \in C^{2+\alpha}$ $((-\infty,-1] \times[0, T])$ follows by the classical Schauder estimates. By repeating differentiating Equation (2.2) in $z$ and applying the same argument, we prove 
that $\mathrm{e}^{-p z} D_{z}^{k} h \in C^{2+\alpha}((-\infty,-1] \times[0, T]), \forall k>0$. Similarly, we show that

$$
h(z, t)=f(x, t)-\tilde{d}(t), \quad z=-\log (1-x), \quad \tilde{d}(t)=f(1, t)
$$

satisfies $\mathrm{e}^{p z} D_{z}^{k} h \in C^{2+\alpha}([1,+\infty) \times[0, T])$. The regularity in $t$ follows from the regularity in $z$ through Equation (2.1). Translating back to the variable $x$, we easily conclude that $f \in C_{s}^{k+\alpha}\left(Q_{T}\right)$, for all $k \geq 2$, as desired.

Combining Theorem 2.1 and Proposition 3.1 gives:

Theorem 3.2. Assume that at time $t=0, \Sigma$ is a surface of revolution $r=$ $f(x, 0)$, with $f(\cdot, 0) \in C_{s}^{2+\alpha, p}(I)$ for some $0<\alpha, p<1$. In addition, assume that $f(\cdot, 0)$ satisfies conditions (1.2)-(1.4). Then, there exists $\tau_{0}>0$ for which the HMCF (1.1) admits a unique solution $f \in C^{\infty, p}\left(Q_{\tau_{0}}\right)$ on $Q_{\tau_{0}}=$ $I \times\left[0, \tau_{0}\right]$, which satisfies conditions (1.2)-(1.5).

We finish this section with the additional observation that the area of the surface of revolution $r=f(x, t)$ remains constant under the HMCF.

Proposition 3.3. Assume that $f \in C^{\infty, p}\left(Q_{T}\right)$ is a solution of the HMCF (1.1) on $Q_{T}$ for some $T>0$, which satisfies conditions (1.2)-(1.5). Then, the area

$$
A(t)=\int_{0}^{1} f \sqrt{1+f_{x}^{2}} d x
$$

of the surface $r=f(x, t)$ remains constant under the flow.

Proof. We will show that

$$
\frac{d A(t)}{d t}=0
$$

by a direct computation. Set $R=\arctan f_{x}$. A direct computation shows that $R$ evolves by

$$
R_{t}=\frac{\left(R_{x x}+R_{x}^{2} \tan R\right) \cos ^{2} R}{\left(1-R_{x} f\right)^{2}}
$$

Hence

$$
\frac{\partial(f \sec R)}{\partial t}=\frac{R_{x x} f \sin R+R_{x} \sec R-R_{x}^{2} f \cos R}{\left(1-R_{x} f\right)^{2}} .
$$

Observing that

$$
\frac{\partial}{\partial x}\left(\frac{\sin R}{1-R_{x} f}\right)=\frac{R_{x x} f \sin R+R_{x} \sec R-R_{x}^{2} f \cos R}{\left(1-R_{x} f\right)^{2}},
$$


we conclude

$$
\frac{d A(t)}{d t}=\frac{d}{d t} \int_{0}^{1} f \sec R d x=\int_{0}^{1} \frac{\partial}{\partial x}\left(\frac{\sin R}{1-R_{x} f}\right) d x .
$$

To conclude the proof, we need to show that

$$
\frac{\sin R}{1-R_{x} f}=0, \quad \text { at } x=0,1 .
$$

To this end, we use the formula

$$
-f f_{x x}+f_{x}^{2}+1=\left(1-R_{x} f\right) \sec ^{2} R
$$

and (1.1) to compute that

$$
\frac{\sin R}{1-R_{x} f}=f\left(f_{t}+\frac{1}{f}\right) \sin R
$$

Since $f_{t}=-1 / f$ at $x_{i}=0,1$, by (1.5), and $\sin R=-\pi / 2$ at $x=0$ and $\sin R=\pi / 2$ at $x=1$, the desired equality follows, finishing the proof.

\section{A priori derivative bounds}

Throughout this section, we will assume that $f \in C^{\infty, p}\left(I \times\left[0, T_{0}\right)\right)$ is a strictly convex positive solution of the HMCF (1.1) with strictly negative mean curvature $H(x, t)<0$ and that it satisfies the non-degeneracy condition

$$
x^{2-p}(1-x)^{2-p} f_{x x}(x, t) \geq c(T)>0, \quad \text { on } Q_{T}=I \times[0, T]
$$

for every $0<T<T_{0}$. We show uniform derivative bounds up to $T_{0}$ as stated next.

Theorem 4.1. Assume that $f \in C^{\infty, p}\left(I \times\left[0, T_{0}\right)\right)$ is a solution of the HMCF (1.1) satisfying

$$
\tilde{H}=-f f_{x x}+f_{x}^{2}+1<0 \quad \text { on } I \times\left[0, T_{0}\right)
$$

and the non-degeneracy condition (4.1). Then, there exist constants $C_{0}<\infty$ and $c_{0}>0$, depending only on the initial data, such that $f$ satisfies the uniform derivative bounds

$$
x^{1-p}(1-x)^{1-p}\left|f_{x}\right| \leq C_{0} \quad \text { and } \quad x^{2-p}(1-x)^{2-p} f_{x x} \leq C_{0}
$$


and the non-degeneracy condition

$$
x^{2-p}(1-x)^{2-p} f_{x x} \geq c_{0}
$$

on $Q_{T_{0}}$.

We begin by estimating $\inf _{Q_{T_{0}}} f$ in terms of $\|f(\cdot, 0)\|_{L^{\infty}(I)}$. This, in particular, shows that a neck-pinch cannot occur.

Proposition 4.2. Under the assumptions of Theorem 4.1, there exists a constant $\beta_{0}>0$, depending only on $\|f(\cdot, 0)\|_{L^{\infty}(I)}$, such that

$$
f(x, t) \geq \beta_{0}, \quad \forall(x, t) \in Q_{T_{0}} .
$$

Proof. Fix $0<t<T_{0}$. The proof only uses that $F=f(\cdot, t)$ is a continuous function on $I=[0,1]$, smooth in its interior, which satisfies inequality (4.2). We will compare $F$ with the minimal surfaces of revolution $\phi(x)=\theta^{-1} \cosh \left(\theta\left(x-x_{0}\right)\right)$ centered at the minimum point $x_{0}$ of $F$. We chose $\theta^{-1}=F\left(x_{0}\right)$ so that $\phi\left(x_{0}\right)=F\left(x_{0}\right)$. We observe that the inequality $\tilde{H}=-f f_{x x}+f_{x}^{2}+1<0$ implies the derivative bound

$$
\frac{d}{d x}\left(\frac{1+F^{\prime 2}}{F^{2}}\right)=\frac{2 F^{\prime}}{F^{3}}\left(F F^{\prime \prime}-F^{\prime 2}-1\right)>0
$$

so that

$$
\left(\frac{F\left(x_{0}\right)}{F(x)}\right)^{2}\left(1+F^{\prime}(x)^{2}\right)>1
$$

provided $x>x_{0}$ (or equivalently $F^{\prime}(x)>0$ ). Hence

$$
\frac{d}{d x}\left(F\left(x_{0}\right) \operatorname{arcosh} \frac{F(x)}{F\left(x_{0}\right)}\right)>1,
$$

which yields the inequality

$$
F(x)>\theta^{-1} \cosh \left(\theta\left(x-x_{0}\right)\right), \quad x_{0}<x<1
$$

since $\theta^{-1}=F\left(x_{0}\right)$. The same argument also gives the inequality for $0<x<x_{0}$, finishing the proof of the proposition.

The following simple first-order derivative upper bound will be used in the sequel. 
Lemma 4.3. Under the assumptions of Theorem 4.1, we have

$$
x(1-x)\left|f_{x}\right| \leq\|f\|_{L^{\infty}\left(Q_{T_{0}}\right)}, \quad \text { on } Q_{T_{0}} .
$$

Proof. We will estimate from above $-x f_{x}$ and $(1-x) f_{x}$ on $Q_{T_{0}}$ using the inequality $-f f_{x x}+f_{x}^{2}+1<0$. Set $X=-x f_{x}(\cdot, t)$, for $0<t<T_{0}$. The maximum of $X$ on $I$ cannot occur at the boundary of $I$, as $X=0$ at $x=0$ and $X<0$ near $x=1$. At an interior maximum point $x_{0}, X_{x}=0$, i.e., $x f_{x x}=-f_{x}$. Hence,

$$
f_{x}^{2}<f f_{x x}=\frac{-f f_{x}}{x}
$$

at $x_{0}$, readily implying that $X<f$ at $x_{0}$. The bound on $(1-x) f_{x}$ can be shown similarly.

Lemma 4.4. Under the assumptions of Theorem 4.1, there exists a constant $C_{0}>0$ such that

$$
x^{1-q}(1-x)^{1-q}\left|f_{x}\right| \leq C_{0}, \quad \text { on } Q_{T_{0}}
$$

for any $0<q<p$.

Proof. Fix $0<q<p$. From the previous lemma, it is enough to establish the bound (4.7) near the boundary points $x_{i}=0,1$. Set $G=-1 / f_{x}$ and fix $T<T_{0}$. We will show that $G \geq c_{0} x^{1-q}$ on $Q_{T}^{\delta}=[0, \delta] \times[0, T]$, for $\delta, c_{0}>0$ sufficiently small, both independent of $T$, by comparing with explicit sub-solutions.

A direct computation shows that $G$ satisfies the evolution equation

$$
G_{t}=\frac{G^{2}\left(1+G^{2}\right) G_{x x}-G\left(1+2 G^{2}\right) G_{x}^{2}}{\left(1+G^{2}-f G_{x}\right)^{2}}
$$

Defining $\phi(x, t)=\alpha(t) x^{1-q}$, one easily computes that

$$
\phi_{t}<\frac{\phi^{2}\left(1+\phi^{2}\right) \phi_{x x}-\phi\left(1+2 \phi^{2}\right) \phi_{x}^{2}}{\left(1+\phi^{2}-f \phi_{x}\right)^{2}},
$$

provided

$$
\alpha^{\prime}(t)<-\frac{\alpha(t)^{3}(1-q)\left((2-q) \alpha(t)^{2} x^{2}+x^{2 q}\right)}{\left(\alpha(t)^{2} x^{2}+x^{2 q}-(1-q) f(x, t) \alpha(t) x^{q}\right)^{2}} .
$$


Since $0<c \leq f(x, t) \leq C$ on $I \times\left[0, T_{0}\right)$, there exist constants $M<\infty$ and $\delta>0$ such that inequality (4.10) holds, provided

$$
\alpha^{\prime}(t)<-M \alpha(t), \quad \text { on } Q_{T}^{\delta}=[0, \delta] \times[0, T]
$$

in particular, if $\alpha(t)=\alpha_{0} \mathrm{e}^{-(M / 2) t}$, with $\alpha_{0}>0$. Hence, $\phi(x, t)=\alpha_{0} \mathrm{e}^{-(M / 2) t}$ $x^{1-q}$ satisfies (4.9). Since $f(\cdot, 0) \in C_{s}^{2+\alpha, p}(I)$, we may choose $\alpha_{0}>0$, sufficiently small, such that $G>\phi$ on $(0,1]$ at $t=0$. Also, by the previous Lemma, $G(\delta, t)>\phi(\delta, t), \forall t<T_{0}$ if $\alpha_{0}>0$ is chosen sufficiently small. Since, in addition $x^{1-p}\left|f_{x}\right| \leq C(T)$ on $Q_{T}^{\delta}$ (because $\left.f \in C^{\infty, p}(I \times[0, T])\right)$, there exists $\mu_{T}$ with $0<\mu_{T}<\delta$ such that $G>\phi$ on $\left(0, \mu_{T}\right] \times[0, T]$. Hence, the classical maximum principle shows that $G>\phi$ on $Q_{T}^{\delta}$. Since $T$ is arbitrary, we conclude that $G \geq c_{0} x^{1-q}$ with $c_{0}=\alpha_{0} \mathrm{e}^{-(M / 2) T_{0}}$ on $[0, \delta] \times\left[0, T_{0}\right)$, implying the desired bound $x^{1-q}\left|f_{x}\right| \leq c_{0}^{-1}$. The bound near the boundary point $x=1$ can be obtained similarly.

We will next establish the following sharp first-order derivative upper bound.

Lemma 4.5. Under the assumptions of Theorem 4.1, there exists a constant $C_{0}>0$ such that

$$
x^{1-p}(1-x)^{1-p}\left|f_{x}\right| \leq C_{0}, \quad \text { on } Q_{T_{0}} .
$$

Proof. We only need to show the bound (4.11) near the boundary points $x_{i}=0,1$, since the interior bound follows from Lemma 4.3. Let us show the bound near $x=0$ by estimating $F=-x^{1-p} f_{x}>0$ from above. The bound near $x=1$ can be shown similarly. Because the equations become non-degenerate when introducing the variable $z=\log x$ (as seen in previous sections), we set

$$
h(z, t)=f(x, t)-d(t), \quad z=\log x, d(t)=f(0, t)
$$

and estimate $F$ in the new variable $z$ namely

$$
G=-\mathrm{e}^{-p z} h_{z}
$$

Fix $T<T_{0}$. We will show that $G \leq C_{0}$ (with $C_{0}$ independent of $T$ ) on $S_{T}^{\delta}=$ $(-\infty,-\delta] \times(0, T)$, provided $\delta$ is sufficiently large. A direct computation 
shows that $G$ satisfies the evolution equation

$$
G_{t}=\alpha(z, t) G_{z z}+\beta_{1}(z, t) G_{z}+\beta_{2}(z, t) G_{z}^{2}+L, \quad \text { on } S_{T}^{\delta}
$$

with

$$
\alpha=\frac{\mathrm{e}^{2 z}+\mathrm{e}^{2 p z} G^{2}}{D^{2}}, \quad \beta_{1}=\frac{-\mathrm{e}^{2 p z} G^{2}+\mathrm{e}^{2 z}(-3+2 p)}{D^{2}}, \quad \beta_{2}=\frac{-\mathrm{e}^{2 p z} G}{D^{2}}
$$

and

$$
D=\mathrm{e}^{2 z}+\mathrm{e}^{2 p z} G^{2}+\mathrm{e}^{p z}\left[G_{z}-(1-p) G\right](h+d) .
$$

The lower order term $L$ is given explicitly in terms of $G$ by

$$
L=\frac{(1-p)\left[\mathrm{e}^{2 p z} G^{2}+(2-p) \mathrm{e}^{2 z}\right]}{\left[\mathrm{e}^{2 z}+\mathrm{e}^{2 p z} G^{2}+\mathrm{e}^{p z}\left[G_{z}-(1-p) G\right](h+d)\right]^{2}} .
$$

Setting $\beta=\beta_{1}+G_{z} \beta_{2}$, we conclude that

$$
G_{t}=\alpha(z, t) G_{z z}+\beta(z, t) G_{z}+L, \quad \text { on } S_{T}^{\delta} .
$$

The coefficients $\alpha, \beta$ satisfy the conditions

$$
\|\alpha\|_{C^{2}\left(S_{T}^{\delta}\right)}+\|\beta\|_{C^{1}\left(S_{T}^{\delta}\right)} \leq \lambda(T, \delta)^{-1}<\infty
$$

and

$$
\alpha(z, t) \geq \lambda(T, \delta)>0, \quad \text { on } S_{T}^{\delta}
$$

In addition,

$$
\|G\|_{C^{\infty}\left(S_{T}^{\delta}\right)} \leq C(T)<\infty
$$

since $f \in C^{\infty, p}(I \times[0, T])$, by assumption. We will use (4.16) and the classical comparison principle to estimate $G$ from above on $S_{T}^{\delta}$. First, choose $\delta$ sufficiently large so that $G(-\delta, t) \leq C_{0}$ on $0<t<T_{0}$ (which is possible from the previous lemma) and also $G(z, 0) \leq C_{0}$ on $(-\infty, \delta)$. Let

$$
M=\frac{8}{(1-p) \beta_{0}^{2}}
$$

with $\beta_{0}$ as in Proposition 4.2 and set $\tilde{G}=G-\left(M t+C_{0}\right)$ with $C_{0}$ chosen, as above, so that $\tilde{G}<0$ on the parabolic boundary of the cylinder $S_{T}^{\delta}$. The 
differential inequality (4.16) implies that

$$
\tilde{G}_{t} \leq \alpha(z, t) \tilde{G}_{z z}+\beta_{1}(z, t) \tilde{G}_{z}+L-M, \quad \text { on } S_{T}^{\delta}
$$

Let

$$
m(t)=\sup \{\tilde{G}(z, t), z \in(-\infty,-\delta]\} .
$$

If $m(t)$ first becomes zero at an interior point $x_{0}(t) \in(-\infty,-\delta)$, then at this point where $\tilde{G}=0$ and $\tilde{G}_{z}=G_{z}=0$, we have

$$
L=\frac{(1-p)\left[\mathrm{e}^{2 p z} G^{2}+(2-p) \mathrm{e}^{2 z}\right]}{\left[\mathrm{e}^{2 z}+\mathrm{e}^{p z} G\left(\mathrm{e}^{p z} G-(1-p)(h+d)\right)\right]^{2}} .
$$

By Proposition $4.2, h+d=f \geq \beta_{0}$. Hence, it follows from the previous lemma that $\mathrm{e}^{2 p z} G^{2}+(2-p) \mathrm{e}^{2 z} \leq 2 \mathrm{e}^{2 p z} G^{2}$ and $\left[\mathrm{e}^{2 z}+\mathrm{e}^{p z} G\left(\mathrm{e}^{p z} G-(1-\right.\right.$ $p)(h+d))]^{2} \geq\left[(1-p) \beta_{0} \mathrm{e}^{p z} G\right]^{2} / 4$ on $S_{T}^{\delta}$, if $\delta$ is chosen sufficiently large, independent of $T$. We conclude that at the maximum point $x_{0}(t)$

$$
L \leq \frac{8}{(1-p) \beta_{0}^{2}}=M
$$

implying that

$$
m^{\prime}(t) \leq \alpha(z, t) \tilde{G}_{z z}+\beta_{1}(z, t) \tilde{G}_{z} \leq 0 .
$$

In the case that $\tilde{G}(\cdot, t)$ achieves it supremum at $z=-\infty$, similar arguments can be applied to show that $m^{\prime}(t) \leq 0$. This is because in that situation, there exists a sequence $z_{i} \rightarrow-\infty$ such that $G\left(z_{i}, t\right) \rightarrow m(t)$ and

$$
\lim _{z_{i} \rightarrow-\infty} \tilde{G}_{z}\left(z_{i}, t\right)=0 \quad \text { and } \quad \lim _{z_{i} \rightarrow-\infty} \tilde{G}_{z z}\left(z_{i}, t\right) \leq 0 .
$$

We conclude from the discussion above that $G \leq M T+C_{0}$ on $S_{T}^{\delta}$, finishing the proof of the lemma.

It is easy to observe (and it is left to the reader) that under the assumptions of Theorem 4.1, the non-degeneracy condition (4.1) implies the lower bound

$$
\liminf _{\left|x-x_{i}\right| \rightarrow 0}\left|x-x_{i}\right|^{1-p}\left|f_{x}\right|(x, t) \geq c(T)>0, \quad \text { on } 0 \leq t \leq T
$$

at $x_{i}=0,1$, for all $0<T<T_{0}$.

Actually, this bound is uniform up to $T_{0}$, as stated next. 
Lemma 4.6. Under the assumptions of Theorem 4.1, there exists a constant $c_{0}>0$ such that

$$
\liminf _{\left|x-x_{i}\right| \rightarrow 0}\left|x-x_{i}\right|^{1-p}\left|f_{x}\right|(x, t) \geq c_{0}, \quad \text { on } 0 \leq t \leq T_{0}
$$

at the boundary points $x_{i}=0,1$.

We will first show that the graph of $f$ cannot turn horizontal at the boundary points $x_{i}=0,1$ at time $T_{0}$.

Lemma 4.7. Under the assumptions of Theorem 4.1, there exist $\eta>0$ and two points $0<X_{1}<X_{2}<1$ such that

$$
f_{x}\left(X_{1}, t\right) \leq-\eta \quad \text { and } \quad f_{x}\left(X_{2}, t\right) \geq \eta, \quad \text { on } 0 \leq t \leq T_{0} .
$$

Proof. It is clear from (4.21) that for all points $X_{i}$ sufficiently close to the boundary points $x_{i}=0,1$, we have

$$
f_{x}\left(X_{1}, t\right) \leq-\eta(T) \quad \text { and } \quad f_{x}\left(X_{2}, t\right) \geq \eta(T), \quad \text { on } 0 \leq t \leq T
$$

for all $0<T<T_{0}$. Hence, we only need to show (4.23) near $t=T_{0}$. To this end, we will construct smooth, strictly convex upper barriers $\psi^{i}(x, t)$ on $Q_{T_{0}}$ with

$$
\psi_{x}^{0}\left(0, T_{0}\right)<0 \quad \text { and } \quad \psi_{x}^{1}\left(1, T_{0}\right)>0
$$

and such that

$$
f \leq \psi^{i}, \quad \text { on } Q_{T_{0}}, \quad f\left(x_{i}, t\right)=\psi^{i}\left(x_{i}, t\right), \quad 0 \leq t<T_{0} .
$$

We will construct $\psi=\psi^{0}$, the construction of $\psi^{1}$ is similar. Let $\theta>0$ and $0<x_{0}<1$ be two constants to be determined in the sequel. We consider the solution

$$
\phi(x)=\frac{\mathrm{e}^{\theta\left(x-x_{0}\right)}+\mathrm{e}^{-\theta\left(x-x_{0}\right)}}{2 \theta}
$$

of $-\phi \phi^{\prime \prime}+\phi^{2}+1=0$, and for $\epsilon>0$, we set

$$
\psi^{\epsilon}(x, t)=\phi(x)-\phi(0)+d(t)+\epsilon
$$

with $d(t)=f(0, t)$. A direct computation shows that $\psi^{\epsilon}$ satisfies

$$
\psi_{t} \geq \frac{\psi_{x x}}{-\psi \psi_{x x}+\psi_{x}^{2}+1}
$$


provided

$$
d^{\prime}(t) \geq \frac{1}{\phi(0)-d(t)-\epsilon} .
$$

Since $d^{\prime}(t)=-1 / d(t)$ (as shown in (1.5)), the last inequality holds, provided $\epsilon<\phi(0)<d(t)$. By definition,

$$
\psi^{\epsilon}(0, t)=d(t)+\epsilon>f(0, t), \quad t \leq T_{0} .
$$

Also, by choosing $\theta$ sufficiently small and $x_{0}>0$ sufficiently close to zero, depending only on $\|f(\cdot, 0)\|_{C^{2+\alpha, p}(I)}$, we can make:

(i) $\psi^{\epsilon}(x, 0)>f(x, 0)$ on $I$,

(ii) $\psi^{\epsilon}(1, t)>f(1, t)$ on $0 \leq t \leq T_{0}$ and

(iii) $\phi(0)<d\left(T_{0}\right) \leq d(t)$ on $0 \leq t \leq T_{0}$.

We conclude, by the maximum principle, that $\psi^{\epsilon}(x, t) \geq f(x, t)$ on $Q_{T_{0}}$. Letting $\epsilon \rightarrow 0$, we obtain the estimate

$$
\psi(x, t) \equiv \phi(x)-\phi(0)+d(t) \geq f(x, t)
$$

The lemma now follows from the observation that

$$
\psi_{x}(0, t)=\phi_{x}(0)=\frac{\mathrm{e}^{-\theta x_{0}}-\mathrm{e}^{\theta x_{0}}}{2}<0
$$

Proof of Lemma 4.6. We will only establish the bound $\liminf _{x \rightarrow 0} x^{1-p}\left|f_{x}\right|$ $(x, t) \geq c_{0}>0$, as the proof of $\liminf _{x \rightarrow 1}(1-x)^{1-p}\left|f_{x}\right| \geq c_{0}>0$ is identical. As in the proof of Lemma 4.5, we introduce

$$
h(z, t)=f(x, t)-d(t), \quad z=\log x, d(t)=f(0, t)
$$

and estimate $F=x^{1-p}\left|f_{x}\right|(x, t)$ in the new variable $z$, namely $G=-\mathrm{e}^{-p z} h_{z}$. Fix $T<T_{0}$. We will show that $G \geq c_{0}$ (with $c_{0}$ independent of $T$ ) on $S_{T}^{\delta}=(-\infty,-\delta] \times(0, T)$, provided $\delta$ is sufficiently large so that $G>0$ on $(-\infty,-\delta] \times\left(0, T_{0}\right)$ and actually $G(-\delta, t) \geq c_{0}>0$ on $0<t<T_{0}$ (this is possible because of the previous lemma).

As in the proof of Lemma 4.5, $G$ satisfies the evolution Equation (4.12) on $S_{T}^{\delta}$, with coefficients given by (4.13) and (4.14) and lower order term $L$ 
given by (4.15) and satisfying $L \geq 0$. Hence,

$$
G_{t} \geq \alpha(z, t) G_{z z}+\beta(z, t) G_{z}, \quad \text { on } S_{T}^{\delta},
$$

with $\alpha$ and $\beta=\beta_{1}+G_{z} \beta_{2}$ satisfying the bounds (4.17) and (4.18). In addition, $G$ satisfies the bound (4.19). Hence, the desired bound $G \geq c_{0}$ on $S_{T}^{\delta}$ follows from the comparison principle (as in the proof in Lemma 4.5) and the initial bound $G(x, 0) \geq c_{0}>0$ on $(-\infty,-\delta)$, provided that $\delta$ is chosen sufficiently large.

The following second-order derivative upper bound implies, in particular, that $f_{x x}$ remains bounded up to $T_{0}$ in the interior of $I$.

Lemma 4.8. Under the assumptions of Theorem 4.1, there exists constant $C_{0}<\infty$ such that

$$
x^{2}(1-x)^{2} f_{x x} \leq C_{0}, \quad \text { on } Q_{T_{0}} .
$$

Proof. Let $T<T_{0}$. We will estimate $F=x^{2}(1-x)^{2} f_{x x}$ on $Q_{T}$ by controlling its evolution. Clearly, $F=0$ at both boundaries $x_{i}=0,1$, and hence we will just need to control $d F_{\max } / d t$ at an interior maximum point. A direct computation shows that $F$ evolves by

$$
F_{t}=\alpha(x, t) F_{x x}+\beta(x, t) F_{x}+L,
$$

with $\alpha \geq 0$ on $Q_{T}$ and $\|\alpha\|_{L^{\infty}\left(Q_{T}\right)}<\infty,\|\beta\|_{L^{\infty}\left(Q_{T}\right)}<\infty$ and

$$
L=\frac{\sum_{i=1}^{4} \gamma_{i} F^{i}}{D^{3}}, \quad D=x^{2}(1-x)^{2}\left(1+f_{x}^{2}-f f_{x x}\right)<0
$$

and $\gamma_{i}$ given by

$$
\gamma_{4}=f, \quad \gamma_{3}=W^{2}+4(1-2 x) f W-x^{2}(1-x)^{2}
$$

and

$$
\gamma_{2}=-2\left[W^{2}+x^{2}(1-x)^{2}\right][2(2 x-1) W-(1-6 x(x-1)) f]
$$

and

$$
\gamma_{1}=2[3+10 x(x-1)]\left[W^{2}+x^{2}(1-x)^{2}\right]^{2},
$$

with $W=x(1-x) f_{x}$ bounded (by Lemma 4.3). 
Claim. There exist a constant $C_{0}$, depending only on the initial data, such that if $F_{\max }(t) \geq C_{0}$, then $d F_{\max } / d t \leq 0$.

Indeed, we only need to show that if $F_{\max }(t) \geq C_{0}$, then $L\left(x_{0}, t\right) \leq 0$ at the interior maximum point $x_{0}$ of $F(\cdot, t)$. Since, $D \leq 0$ and $\gamma_{4}=f \geq \beta_{0}>0$, by Proposition 4.2 , this easily follows by observing that the $\gamma_{i}, i=1,2,3$, are bounded.

We will next show the optimal second-order derivative upper bound.

Lemma 4.9. Under the assumptions of Theorem 4.1, there exists constant $C_{0}<\infty$ such that

$$
x^{2-p}(1-x)^{2-p} f_{x x} \leq C_{0}, \quad \text { on } Q_{T_{0}} .
$$

Proof. We only need to show the bound (4.37) near the boundary points $x_{i}=0,1$, since the interior bound follows from Lemma 4.8. Let us show the bound near $x=0$ by estimating $F=x^{2-p} f_{x x}$ from above. The bound near $x=1$ can be shown similarly. Because the equations become non-degenerate when introducing the variable $z=\log x$ (as seen in previous sections), it is simpler to set

$$
h(z, t)=f(x, t)-d(t), \quad z=\log x, d(t)=f(0, t)
$$

and estimate $F$ in the new variable $z$, namely

$$
G=\mathrm{e}^{-p z}\left(h_{z z}-h_{z}\right)
$$

Fix $T<T_{0}$. We will show that $G \leq C_{0}$ (with $C_{0}$ independent of $T$ ) on $S_{T}^{\delta}=$ $(-\infty,-\delta] \times(0, T)$, provided $\delta$ is sufficiently large. A direct computation shows that $G$ satisfies the evolution equation

$$
G_{t}=\alpha(z, t) G_{z z}+\beta_{1}(z, t) G_{z}+\beta_{2}(z, t) G_{z}^{2}+L
$$

on $S_{T}^{\delta}$, with

$$
\begin{aligned}
& \alpha=\frac{\mathrm{e}^{2 z}+\mathrm{e}^{2 p z} W^{2}}{D^{2}}, \quad \beta_{2}=\frac{2 \mathrm{e}^{p z}\left(\mathrm{e}^{2 z}+\mathrm{e}^{2 p z} W^{2}\right)(h+d)}{D^{3}} \\
& D=\mathrm{e}^{2 z}+\mathrm{e}^{2 p z} W^{2}-\mathrm{e}^{p z} G(h+d)<0, \quad W=-\mathrm{e}^{-p z} h_{z}
\end{aligned}
$$


and

$$
\begin{aligned}
\beta_{1}= & \frac{G(h+d)\left[(2 p-3) \mathrm{e}^{(2+p) z}+\mathrm{e}^{3 p z} W(2 G+(2 p-3) W)\right]}{D^{3}} \\
& +\frac{\left[\mathrm{e}^{2 z}+\mathrm{e}^{2 p z} W^{2}\right]\left[\mathrm{e}^{2 z}(2 p-5)+\mathrm{e}^{2 p z} W(2 G+(2 p-5) W)\right]}{D^{3}} .
\end{aligned}
$$

The lower order term $L$ is of the form

$$
L=\frac{\sum_{i=1}^{4} \gamma_{i} G^{i}}{D^{3}}
$$

with

$$
\begin{gathered}
\gamma_{4}=\mathrm{e}^{3 p z}(h+d), \quad \gamma_{3}=\mathrm{e}^{3 p z}\left[\mathrm{e}^{p z} W^{2}-2(2-p) W(h+d)-\mathrm{e}^{(2-p) z}\right] \\
\gamma_{2}=(2-p) \mathrm{e}^{3 p z}\left(\mathrm{e}^{2(1-p) z}+W^{2}\right)\left[(1-p)(h+d)-2 \mathrm{e}^{p z} W\right] \\
\gamma_{1}=\left(6-5 p+p^{2}\right)\left(\mathrm{e}^{2 z}+\mathrm{e}^{2 p z} W^{2}\right)^{2}
\end{gathered}
$$

Notice that by Lemma $4.5, W \leq C_{0}$ on $S_{T}^{\delta}, \forall T<T_{0}$.

We may write (4.29) in the form

$$
G_{t}=\alpha(z, t) G_{z z}+\beta(z, t) G_{z}+L, \quad \text { on } S_{T}^{\delta}
$$

with $\beta=\beta_{1}+\beta_{2} G_{z}$.

The coefficients $\alpha, \beta$ satisfy the conditions

$$
\|\alpha\|_{C^{2}\left(S_{T}^{\delta}\right)}+\|\beta\|_{C^{1}\left(S_{T}^{\delta}\right)} \leq \lambda(T, \delta)^{-1}<\infty
$$

and

$$
\alpha(z, t) \geq \lambda(T, \delta)>0, \quad \text { on } S_{T}^{\delta}
$$

In addition, $\|G\|_{C^{\infty}\left(S_{T}^{\delta}\right)} \leq C(T, \delta)<\infty$, since $f \in C^{\infty, p}\left(I \times\left[0, T_{0}\right)\right.$ by assumption in this section. Since $h+d=f \geq \beta_{0}$, by Proposition 4.2, one easily concludes that $\Sigma_{i} \gamma_{i} G^{i}>0$, on $S_{T}^{\delta}$, provided $G \geq M_{0}$ is sufficiently large, depending only on $C_{0}$. Also, $D<0$ on $S_{T}^{\delta}$, provided $G \geq M_{0}$ is sufficiently large. Hence, $L<0$. The lemma now follows from the interior bound of Lemma 4.8 and the comparison principle on $S_{T}^{\delta}$. 
We next bound from below, away from zero, the

$$
F=\frac{x^{2-p}(1-x)^{2-p} f_{x x}}{\left[x^{1-p}(1-x)^{1-p}\left|f_{x}\right|\right]^{1+1 /(1-p)}}=\frac{f_{x x}}{\left|f_{x}\right|^{1+1 /(1-p)}}
$$

as stated in the following lemma. This, combined with Lemma 4.6, will show that $x^{2-p}(1-x)^{2-p} f_{x x}$ is bounded from below away from zero on $Q_{T_{0}}$. The reason for estimating the quotient (4.36) is that we cannot bound $x^{2-p}(1-x)^{2-p} f_{x x}$ from below away from zero directly from its evolution.

Lemma 4.10. Under the assumptions of Theorem 4.1, there exists a constant $c_{0}>0$ such that

$$
F=\frac{x^{2-p}(1-x)^{2-p} f_{x x}}{\left[x^{1-p}(1-x)^{1-p}\left|f_{x}\right|\right]^{1+1 /(1-p)}} \geq c_{0}, \quad \text { on } Q_{T_{0}} .
$$

Proof. We begin by noticing that

$$
F \geq c(J)>0, \quad \text { on } J \times\left[0, T_{0}\right), \quad J \subset \subset I
$$

since $f_{x x} \geq\left(1+f_{x}^{2}\right) / f$ (implied by inequality $\left.-f f_{x x}+f_{x}^{2}+1<0\right)$ and $\left|f_{x}\right| \leq C(J)<\infty$ on $J \times\left[0, T_{0}\right)$ by Lemma 4.3. Since, we only need to estimate $F$ from below near the boundary points, $x_{i}=0,1$.

Let us estimate $\tilde{F}=\left(x^{2-p} f_{x x}\right) /\left(x^{1-p}\left|f_{x}\right|\right)^{1+1 /(1-p)}$ near $x=0$. As in the proof of Lemma 4.6, we will express $\tilde{F}$ in terms of $h(z, t)=f(x, t)-d(t)$, with $z=\log x$, and therefore compute the evolution of

$$
G=\frac{\mathrm{e}^{-p z}\left(h_{z z}-h_{z}\right)}{W^{1+1 /(1-p)}}
$$

with $W=-\mathrm{e}^{-p z} h_{z}>0$ on $S_{T_{0}}^{\delta}=(-\infty,-\delta] \times\left(0, T_{0}\right)$ (choosing $\delta>0$ sufficiently large). By our discussion above,

$$
G(\delta, t) \geq c_{0}>0, \quad \text { on } 0 \leq t \leq T_{0} .
$$

A direct computation shows that $G$ satisfies the evolution equation

$$
G_{t}=\alpha(z, t) G_{z z}+\beta(z, t) G_{z}+L_{1}+L_{2}
$$

with

$$
L_{1}=\frac{\mathrm{e}^{p z}\left[W^{4}-\left(1+3 p-2 p^{2}\right) \mathrm{e}^{(2-p) z} W^{2}+(2-p) \mathrm{e}^{(1-p) z}\right] G^{3}}{(1-p)^{2} D^{3}}
$$


and

$$
L_{2}=\frac{W^{1+1 /(1-p)}\left[\left(6-7 p+2 p^{2}\right) \mathrm{e}^{2(1-p) z}+W^{2}\right] h G^{4}}{(1-p)^{2} D^{3}}
$$

with

$$
D=\mathrm{e}^{(2-p) z}+\mathrm{e}^{p z} W^{2}-G W^{1+1 /(1-p)}(h+d(t))<0, \quad \text { on } Q_{T_{0}},
$$

and coefficients $\alpha, \beta$ which satisfy conditions (4.34) and (4.35), provided $h \in C^{\infty, p}\left(S_{T}^{\delta}\right)$, as assumed.

Claim. There exists a uniform constant $M<\infty$ such that

$$
L_{1}+L_{2} \geq-M G, \quad \text { on } S_{T}^{\delta}, \forall T<T_{0},
$$

provided

$$
D<-\frac{1}{2} G W^{1+1 /(1-p)}(h+d(t)) .
$$

Indeed, we observe first that since $W \geq c_{0}>0$ on $Q_{T_{0}}$ (by Lemma 4.6) and $D<0$, we have

$$
L_{1}+L_{2} \geq-C\left(\frac{\mathrm{e}^{p z} W^{4}}{G}+W^{3+1 /(1-p)}\right) \frac{G^{4}}{|D|^{3}}
$$

for a uniform constant $C$. Observing that $W^{3+1 /(1-p)} \geq c_{0} \mathrm{e}^{p z} W^{4} / G$ (with $c_{0}>0$ uniform on $Q_{T_{0}}$ ) is equivalent to $f_{x x} \geq \tilde{c}_{0} f_{x}^{2}$, which is implied by the inequality $-f f_{x x}+f_{x}^{2}+1<0$, we conclude that

$$
L_{1}+L_{2} \geq-C \frac{W^{3+1 /(1-p)} G^{4}}{|D|^{3}}
$$

for a uniform constant $C$. Hence, assumption (4.41) and Lemmas 4.5 and 4.6 imply (4.40).

We will use this inequality and the comparison principle to control $G$ from below on $S_{T_{0}}^{\delta}$ for $\delta>0$ sufficiently small. Let $\zeta(t)=\zeta_{0} \mathrm{e}^{-2 M t}$ be the solution of $\zeta^{\prime}(t)=-2 M \zeta(t)$, with $\zeta_{0}$ sufficiently small so that $G(\cdot, 0) \geq \zeta_{0}$ on $z=\log x<0$. Next, choose $\delta>0$ sufficiently small so that (4.38) and (4.41) hold, provided $G(z, t) \geq \zeta(t)=\zeta_{0} \mathrm{e}^{-2 M t}$. This is possible since $W$ is bounded from above and below away from zero on $S_{T_{0}}^{\delta}$. Let $T<T_{0}$. Since $h \in C^{\infty, p}\left(S_{T}^{\delta}\right)$ and $f$ satisfies condition (4.1), $G$ and all its derivatives are bounded in $S_{T}^{\delta}$. The comparison principle then implies that $G \geq \zeta(t)$ on $S_{T}^{\delta}$, which readily implies the bound $G \geq \zeta_{0} \mathrm{e}^{-2 M T_{0}}$ on $S_{T_{0}}^{\delta}$, finishing the proof of the Lemma. 


\section{Existence and regularity up to when $H=0$}

In this section, will show that under the initial conditions of Theorem 2.1, the solution $f$ of the HMCF (1.1) exists for as long as the mean curvature $H$ remains strictly negative, as stated in the next result.

Theorem 5.1. Under the assumptions of Theorem 2.1 the solution $f$ of the HMCF (1.1) exists up to the time $T_{0}$, where first $H\left(x_{0}, T_{0}\right)=0$, at some point $x_{0} \in[0,1]$. In addition, $f \in C^{2, p}\left(Q_{T_{0}}\right)$ and it satisfies the nondegeneracy condition

$$
x^{2-p}(1-x)^{2-p} f_{x x} \geq c_{0}>0, \quad \text { on } Q_{T_{0}} .
$$

The proof of Theorem 5.1 readily follows from the next result.

Lemma 5.2. Assume that $f \in C_{s}^{2+\alpha, p}\left(Q_{\tau}\right)$ is a positive strictly convex solution of the $H M C F(1.1)$ on $I \times[0, T)$, which satisfies the non-degeneracy condition

$$
x^{2-p}(1-x)^{2-p} f_{x x}(x, t) \geq c(\tau)>0, \quad \text { on } Q_{\tau},
$$

for all $\tau<T$ and the condition

$$
\tilde{H}=-f f_{x x}+f_{x}^{2}+1<-\delta_{0}<0, \quad \text { on } I \times[0, T) .
$$

Then, the solution $f$ can be extended to exist on $Q_{\tilde{T}}$ for some $\tilde{T}>T$. In addition, $f \in C^{\infty, p}\left(Q_{\tilde{T}}\right)$, and it satisfies the non-degeneracy condition (5.2) on $Q_{\tilde{T}}$.

In the proof of Lemma 5.2, we will use the following variant of the Krylov-Safonov [6] Hölder regularity result.

Proposition 5.3. Let $u$ be $a C^{2}$ solution of the semi-linear equation

$$
u_{t}=\alpha(z, t) u_{z z}+\beta_{1}(z, t) u_{z}+\beta_{2}(z, t) u_{z}^{2}+L(z, t)
$$

on $Q_{2}=[-2,2] \times[-4,0]$, with coefficients satisfying $\beta_{2} \leq 0$ and

$$
\|\alpha\|_{L^{\infty}\left(Q_{2}\right)}+\left\|\beta_{1}\right\|_{L^{\infty}\left(Q_{2}\right)}+\left\|\beta_{2}\right\|_{L^{\infty}\left(Q_{2}\right)}+\|L\|_{L^{\infty}\left(Q_{2}\right)} \leq \lambda^{-1}
$$

and also $\alpha(z, t) \geq \lambda$ on $Q_{2}$, for some number $\lambda>0$. Assume, in addition, that

$$
0<\mu \leq u(z, t) \leq M<\infty, \quad \text { on } Q_{2} \text {. }
$$


Then, there exists a number $\theta \in(0,1)$ and a constant $C(\lambda, \mu, M)<\infty$ such that

$$
\|u\|_{C^{\theta}\left(Q_{1}\right)} \leq C(\lambda, \mu, M), \quad \text { on } Q_{1}=[-1,1] \times[-1,0] .
$$

Proof. For a point, $\left(x_{0}, t_{0}\right) \in Q_{1}$ and $\rho<1$, and let us denote by $m_{\rho}=$ $\inf _{Q_{\rho}\left(x_{0}, t_{0}\right)} u, M_{\rho}=\sup _{Q_{\rho}\left(x_{0}, t_{0}\right)} u$. Also, to simplify the notation, let us set $Q_{\rho}:=Q_{\rho}\left(x_{0}, t_{0}\right)$ and $Q_{\rho}^{-}:=Q_{\rho}\left(x_{0}, t_{0}-3 \rho^{2} / 4\right)$.

Claim. There exist absolute constants $0<\gamma, \eta<1$ and a constant $C=$ $C\left(\lambda,\|u\|_{L^{\infty}\left(Q_{2}\right)}\right)$ such that for all $\rho>0$, sufficiently small, we have

$$
\operatorname{osc}_{Q_{\rho / 4}} u \leq \gamma \operatorname{osc}_{Q_{\rho}} u+C \rho^{1 / 2}
$$

We begin by observing that since $\beta_{2} \leq 0$, the function $v=u-m_{\rho} \geq 0$ is a sub-solution of equation

$$
v_{t} \leq \alpha(z, t) v_{z z}+\beta_{1}(z, t) v_{z}+L(z, t)
$$

on $Q_{2}=[-2,2] \times[-4,0]$. Hence, the local maximum principle for subsolutions to linear parabolic equations [7, Theorem 7.26] implies the bound

$$
\sup _{Q_{\rho / 4}^{-}}\left(u-m_{\rho}\right) \leq C(\lambda, p)\left\{\left(\rho^{-3} \int_{Q_{\rho / 2}^{-}}\left(u-m_{\rho}\right)^{p} d x d t\right)^{1 / p}+\rho^{1 / 2}\|L\|_{L^{2}\left(Q_{2}\right)}\right\}
$$

for any $p>0$. Since with $\|L\|_{L^{\infty}\left(Q_{2}\right)} \leq \lambda^{-1}$, we conclude the estimate

$$
\sup _{Q_{\rho / 4}^{-}}\left(u-m_{\rho}\right) \leq C(\lambda, p)\left\{\left(\rho^{-3} \int_{Q_{\rho / 2}^{-}}\left(u-m_{\rho}\right)^{p} d x d t\right)^{1 / p}+\rho^{1 / 2}\right\}
$$

For a number $d>0$, set $w=\sqrt{u} / d>0$ on $Q_{\rho}$. A direct computation shows that $\mathrm{w}$ is a solution of the equation

$$
w_{t}=\alpha(z, t) w_{z z}+\beta_{1}(z, t) w_{z}+\tilde{\beta}_{2}(z, t) w_{z}^{2}+\frac{L(z, t)}{2 d \sqrt{u}}
$$

on $Q_{\rho}$, with

$$
\tilde{\beta}_{2}(z, t)=2 d \beta_{2}(z, t) w(z, t)+\frac{\alpha(z, t)}{w(z, t)} .
$$

Although $\beta_{2}<0$, the bounds on the coefficients $\alpha$ and $\beta_{2}$ imply that we can make $\tilde{\beta}_{2} \geq 0$ if

$$
d=\frac{\lambda^{2}}{2 M^{2}}
$$


i.e., depending only on $\lambda$ and $M$. Hence, $w$ is a super-solution of the equation

$$
w_{t} \geq \alpha(z, t) w_{z z}+\beta_{1}(z, t) w_{z}+\tilde{L}
$$

on $Q_{\rho}$, with

$$
\|\tilde{L}\|_{L^{\infty}\left(Q_{2}\right)}=\left\|\frac{L(z, t)}{2 d \sqrt{u}}\right\|_{L^{\infty}\left(Q_{2}\right)} \leq \frac{\sqrt{\mu}^{-1}\|L\|_{L^{\infty}\left(Q_{2}\right)}}{2 d}
$$

by (5.3). Hence, the Harnack estimate for super-solutions to linear parabolic equations [7, Theorem 7.22] implies the bound

$$
\begin{aligned}
& \left(\rho^{-3} \int_{Q_{\rho / 2}^{-}}\left(\sqrt{u}-\sqrt{m_{\rho}}\right)^{p_{0}} d x d t\right)^{1 / p_{0}} \\
& \quad \leq C(\lambda)\left(\inf _{Q_{\rho / 2}}\left(\sqrt{u}-\sqrt{m_{\rho}}\right)+d \rho^{1 / 2}\|\tilde{L}\|_{L^{2}\left(Q_{2}\right)}\right)
\end{aligned}
$$

for some $p_{0}>0$. However, by (5.3), we have

$$
\frac{u-m_{\rho}}{2 \sqrt{M}} \leq \sqrt{u}-\sqrt{m_{\rho}}=\frac{u-m_{\rho}}{\sqrt{u}+\sqrt{m_{\rho}}} \leq \frac{u-m_{\rho}}{2 \sqrt{\mu}},
$$

which combined with (5.7) and (5.6) gives

$$
\left(\frac{1}{\left|Q_{\rho / 2}^{-}\right|} \int_{Q_{\rho / 2}^{-}}\left(u-m_{\rho}\right)^{p_{0}} d x d t\right)^{1 / p_{0}} \leq C(\lambda, \mu, M)\left(\inf _{Q_{\rho / 2}}\left(u-m_{\rho}\right)+\rho^{1 / 2}\right)
$$

for some $p_{0}>0$. Combining (5.5) with $p=p_{0}$ and (5.8), we obtain

$$
\sup _{Q_{\rho / 4}^{-}}\left(u-m_{\rho}\right) \leq C(\lambda, \mu, M)\left(\inf _{Q_{\rho / 2}}\left(u-m_{\rho}\right)+\rho^{1 / 2}\right) .
$$

Similarly, we obtain the estimate

$$
\sup _{Q_{\rho / 4}^{-}}\left(M_{\rho}-u\right) \leq C(\lambda, \mu, M)\left(\inf _{Q_{\rho / 2}}\left(M_{\rho}-u\right)+\rho^{1 / 2}\right) .
$$

Combining (5.9) and (5.10) in a standard way implies the Claim from which the Hölder continuity of $u$ follows. For details, we refer the reader to [7]. 
Proof of Lemma 5.2. We begin by observing that by Theorem 4.1, the solution $f$ satisfies the uniform derivative bounds (4.3) and (4.4) on $Q_{T}$. We will use these bounds to show that

$$
\|f\|_{C_{s}^{2+\theta, p}\left(Q_{T}\right)} \leq C_{0}<\infty, \quad \text { on } Q_{T}
$$

for some number $0<\theta<1$, with $c_{0}, C_{0}$ independent of $T<T_{0}$. Since, in addition, $\tilde{H}=-f f_{x x}+f_{x}^{2}+1 \leq-\delta_{0}<0$ at $t=T$, (5.11) combined with the short-time existence Theorem 2.1 implies that $f$ can be extended to a solution of (1.1) on $Q_{\tilde{T}}$, for some $\tilde{T}>T$, with $f \in C_{s}^{2+\theta, p}\left(Q_{\tilde{T}}\right)$ and satisfying conditions $(1.2)-(1.4)$ on $Q_{\tilde{T}}$. Theorem 3.2 then implies that $f \in C^{\infty, p}\left(Q_{\tilde{T}}\right)$, finishing the proof of the lemma.

To show (5.11), we will prove that $\mathrm{e}^{-p z} h \in C^{2+\theta}\left(S_{T}^{-}\right)$, with $h(z, t)=$ $f(x, t)-f(0, t), z=\log x$, and $S_{T}^{-}=(-\infty, 0] \times[0, T]$ (and similarly e ${ }^{p z} \hat{h} \in$ $C^{2+\theta}\left(S_{T}^{+}\right)$, with $\hat{h}(z, t)=f(x, t)-f(1, t), \quad z=-\log (1-x)$, and $S_{T}^{-}=$ $[0,+\infty) \times[0, T])$.

Set $G=\mathrm{e}^{-p z}\left(h_{z z}-h_{z}\right)=x^{2-p} f_{x x}$. We will show that $G \in C^{\theta}\left(S_{T}^{-}\right)$. As we have shown in the proof of Lemma $4.9, G$ satisfies the equation

$$
G_{t}=\alpha G_{z z}+\beta_{1} G_{z}^{2}+\beta_{2} G_{z}+L
$$

coefficients given by (4.30)-(4.32). It follows from the derivative bounds (4.3) and (4.4) on $Q_{T}$ that $\alpha, \beta_{i}, i=1,2$, and $L$ satisfy the bounds

$$
\|\alpha\|_{L^{\infty}\left(S_{T}^{-}\right)}+\left\|\beta_{i}\right\|_{L^{\infty}\left(S_{T}^{-}\right)}+\|L\|_{L^{\infty}\left(S_{T}^{-}\right)} \leq \lambda^{-1}
$$

and also $\alpha(z, t) \geq \lambda$ on $S_{T}^{-}$, for a positive constant $\lambda>0$. Since, in addition, $0<c_{0} \leq G \leq C_{0}<\infty$ on $S_{T_{0}}^{-}$, we conclude from Proposition 5.3 that $\|G\|_{C^{\theta}\left(S_{T}^{-}\right)}<C_{0}(\lambda)<\infty$. Similarly, from the evolution of $W=-\mathrm{e}^{-p z} h_{z}$, the derivative bounds (4.3), (4.4) and Proposition 5.3, it follows that $\|W\|_{C^{\theta}\left(S_{T}^{-}\right)}<C_{0}(\lambda)<\infty$. Combining the two implies the desired bound $\left\|\mathrm{e}^{-p z} h\right\|_{C^{2, \theta}\left(S_{T}^{-}\right)}<C_{0}<\infty$, for some constant $C_{0}$ independent of $\mathrm{T}$, finishing the proof of the lemma.

We have actually shown Corollary 5.4 which will be used in the next section.

Corollary 5.4. Under the assumptions of Theorem 5.1, there exists a number $\delta>0$ such that $f \in C^{\infty, p}\left(([0, \delta] \cup[1-\delta, 1]) \times\left[0, T_{0}\right]\right)$.

Proof of Theorem 5.1. The theorem follows for the previous lemma and the observation that the bound (5.11) is independent of $T$, for all $T<T_{0}$. 


\section{The blow-up of $\tilde{H}^{-1}$}

In this final section, we will show that

$$
\tilde{H}^{-1}=\frac{1}{-f f_{x x}+f_{x}^{2}+1}
$$

vanishes on an interval of sufficiently large length, depending on the initial data, leading to the proof of Theorem 1.1.

The evolution of $R=\arctan f_{x}$. To simplify the notation, we introduce $R=\arctan f_{x}$. A direct computation shows that $R$ evolves by

$$
R_{t}=\frac{\cos ^{2} R\left(R_{x x}+R_{x}^{2} \tan R\right)}{\left(R_{x} f-1\right)^{2}} .
$$

The evolution of $\tilde{H}$. Using the evolution equations of $f$ and $R$, we find, after a direct calculation, that $\tilde{H}=-f f_{x x}+f_{x}^{2}+1$ evolves by

$$
\begin{aligned}
\tilde{H}_{t}= & \frac{\sec ^{2} R}{\tilde{H}^{2}} \tilde{H}_{x x}-\frac{\sec ^{2} R \sin (2 R)}{\tilde{H} f} \tilde{H}_{x}-\frac{2 \sec ^{2} R}{\tilde{H}^{3}} \tilde{H}_{x}^{2} \\
& +\frac{(1-\tilde{H})(\tilde{H} \cos (2 R)+H-2) \sec ^{2} R}{\tilde{H} f^{2}} .
\end{aligned}
$$

This is an equation of fast-diffusion near $\tilde{H}=0$.

We wish to estimate $\tilde{H}^{-1}$ from above. It is more convenient to estimate

$$
w=-\frac{\sec ^{2} R}{\tilde{H}}=-\frac{1+f_{x}^{2}}{-f f_{x x}+f_{x}^{2}+1}>0
$$

instead. Observe that the estimates of Section 4 imply that

$$
w\left(x_{i}, t\right)=0, \quad \text { at } x_{i}=0,1,0 \leq t \leq T_{0} .
$$

The evolution of $w$. Using the evolution equations of $R$ and $\tilde{H}$, we find, after a direct calculation, that $w$ evolves by the simple equation

$$
w_{t}=w^{2}\left\{w_{x x}-\frac{2 \tan R}{f} w_{x}+\frac{\sec ^{2} R}{f^{2}} w+\frac{2 \sec R}{f^{2}}+\frac{1}{f^{2} w}\right\} .
$$

We will use this equation together with Equations (6.1) and (1.1) to estimate from below the length of the blow-up set of $w$. The simpler model equation

$$
w_{t}=w^{2} w_{x x}+w^{3}
$$


has been studied by Gage and Hamilton [8], Friedman and McLeod [9] and Gage [10]. There, an estimate on the size of the blow-up set was given. Here, we will follow some of the techniques in [10] adopted to our, more complex, equation. Following [8], we define, for $l \in(0,1)$,

$$
w_{l}^{*}(t)=\sup \{b \mid w(x, t)>b \text { on some interval of length } l\} .
$$

The main result of this section states as follows.

Theorem 6.1. Assume that $f \in C^{2, p}\left(Q_{T}\right)$ is a solution of the HMCF (1.1) on $I \times[0, T)$, with $\tilde{H}=-f f_{x x}+f_{x}^{2}+1<0$ on $I \times[0, T)$ and which satisfies the non-degeneracy condition

$$
x^{2-p}(1-x)^{2-p} f_{x x}(x, t) \geq c(T)>0, \quad \text { on } Q_{T} .
$$

Assume, in addition, that $f \in C^{\infty, p}\left(\left(\left[0, \delta_{0}\right] \cup\left[1-\delta_{0}, 1\right]\right) \times[0, T]\right)$ for some $\delta_{0}>0$. Then, there exists an $l_{0}>0$, depending only on the initial data $f(\cdot, 0)$, such that if

$$
\sup _{t \in[0, T)} w_{l_{0}}^{*}(t)<\infty
$$

then $w=-\left(1+f_{x}^{2}\right) \tilde{H}^{-1}$ is bounded on $Q_{T}$.

Our main result Theorem 1.1 stated in the Introduction readily follows from Theorems 6.1 and 5.1 and Corollary 5.4. The proof of Theorem 6.1 will be done in several steps. We begin by observing that the assumptions of Theorem 6.1 imply that there exists a number $\delta>0$ sufficiently small such that

$$
f \in C^{\infty, p}\left(\tilde{Q}^{\delta}\right) \quad \text { and } \quad w \leq 1, \quad \text { on } \tilde{Q}^{\delta}=([0, \delta] \cup[1-\delta, 1]) \times[0, T]
$$

This, in particular, implies that the blow-up set of $w$ is contained in $[\delta, 1-\delta]$.

Lemma 6.2. Assume that $f \in C^{2, p}\left(Q_{T}\right) \cap C^{\infty, p}\left(\tilde{Q}^{\delta}\right)$ and satisfies the nondegeneracy condition (6.5). Then, the function $w$ defined by (6.2) satisfies the integral bound

$$
\int_{0}^{T} \int_{0}^{1} w(x, t) d x d t<\infty
$$


Proof. Choose $\delta>0$ sufficiently small so that (6.7) holds. Using Equation (6.4) and integration by parts on $[\delta, 1-\delta]$, we find

$$
\begin{aligned}
-\frac{d}{d t} \int_{\delta}^{1-\delta} \frac{1}{w} d x= & \int_{\delta}^{1-\delta} \frac{w_{t}}{w^{2}} d x=\int_{\delta}^{1-\delta}\left\{\left[\left(\frac{2 \tan R}{f}\right)_{x}+\frac{\sec ^{2} R}{f^{2}}\right] w\right. \\
& \left.+\frac{2 \sec R}{f^{2}}+\frac{1}{f^{2} w}\right\} d x+B_{\delta}(t)
\end{aligned}
$$

with

$$
B_{\delta}(t)=\left.w_{x}\right|_{\delta} ^{1-\delta}+\left.\frac{2 \tan R}{f} w\right|_{\delta} ^{1-\delta}
$$

Since,

$$
\left(\frac{2 \tan R}{f}\right)_{x}=\frac{2}{f^{2}}+\frac{2 \sec R}{w f^{2}}
$$

by direct computation, we obtain

$$
-\frac{d}{d t} \int_{\delta}^{1-\delta} \frac{1}{w} d x=\int_{\delta}^{1-\delta}\left[\frac{2+\sec ^{2} R}{f^{2}} w+\frac{4 \sec R}{f^{2}}+\frac{1}{f^{2} w}\right] d x+B_{\delta}(t) .
$$

Integrating in time and deleting positive terms give

$$
\int_{0}^{T} \int_{\delta}^{1-\delta} \frac{2+\sec ^{2} R}{f^{2}} w d x d t \leq \int_{\delta}^{1-\delta} \frac{1}{w} d x_{\mid t=0}-\int_{0}^{T} B_{\delta}(t) d t .
$$

Observe next that by (6.7), $\left|B_{\delta}(t)\right| \leq B_{\delta}$ is uniformly bounded on $[0, T]$. Since $\int_{\delta}^{1-\delta} 1 / w d x_{\mid t=0} \leq C_{\delta}<\infty$, depending only on the initial data, we conclude the estimate

$$
\int_{0}^{T} \int_{\delta}^{1-\delta} w d x d t \leq \frac{C(\delta, T)}{\|f\|_{L^{\infty}\left(Q_{T}\right)}}<\infty
$$

which combined with (6.7) implies the desired integral bound.

Lemma 6.3. Under the assumptions of Theorem 6.1, if $\sup _{t \in[0, T)} w_{l_{0}}^{*}(t)<$ $\infty$, then

$$
\sup _{t \in[0, T)} \int_{0}^{1}(\log w)^{+}(x, t) d x<\infty
$$


Proof. Let $\delta>0$ be as in (6.7). We will show that

$$
\sup _{t \in[0, T)} \int_{\delta}^{1-\delta} \log w(x, t) d x<\infty,
$$

provided (6.6) holds. Since, $w \leq 1$ on $[0, \delta] \cup[1-\delta, 1]$ and

$$
w \geq c_{\delta}>0, \quad \text { on }[\delta, 1-\delta] \times[0, T]
$$

(because $f \in C^{2, p}\left(Q_{T}\right)$ ), (6.12) readily implies (6.8).

We begin by expressing (6.4) in the form

$$
(\log w)_{t}=w w_{x x}-\frac{2 \tan R}{f} w w_{x}+\frac{\sec ^{2} R}{f^{2}} w^{2}+\frac{2 \sec R}{f^{2}} w+\frac{1}{f^{2}} .
$$

Since

$$
\frac{2 \tan R}{f} w w_{x}=\left(\frac{\tan R}{f} w^{2}\right)_{x}-\left(\frac{\tan R}{f}\right)_{x}
$$

and

$$
\left(\frac{\tan R}{f}\right)_{x}=\frac{1}{f^{2}}+\frac{\sec R}{w f^{2}}
$$

(by direct computation using (6.1) and (6.2)), we may express (6.14) as (6.15)

$$
(\log w)_{t}=w w_{x x}-\left(\frac{\tan R}{f} w^{2}\right)_{x}+\frac{1+\sec ^{2} R}{f^{2}} w^{2}+\frac{3 \sec R}{f^{2}} w+\frac{1}{f^{2}} .
$$

Integrating by parts Equation (6.15) on $[\delta, 1-\delta]$, we obtain

$$
\begin{aligned}
\frac{d}{d t} \int_{\delta}^{1-\delta}(\log w)_{t}= & -\int_{\delta}^{1-\delta} w_{x}^{2} d x+\int_{\delta}^{1-\delta} \frac{1+\sec ^{2} R}{f^{2}} w^{2} d x \\
& +\int_{\delta}^{1-\delta} \frac{3 \sec R}{f^{2}} w d x+\int_{\delta}^{1-\delta} \frac{1}{f^{2}} d x \\
& +\left.w w_{x}\right|_{\delta} ^{1-\delta}-\left.\frac{\tan R}{f} w^{2}\right|_{\delta} ^{1-\delta} .
\end{aligned}
$$

Observe next that since $f \geq c>0$ on $Q_{T}$ and (6.7) holds, it follows from Proposition 3.1 that

$$
\int_{\delta}^{1-\delta} \frac{1}{f^{2}} d x+\left.w w_{x}\right|_{\delta} ^{1-\delta}-\left.\frac{\tan R}{f} w^{2}\right|_{\delta} ^{1-\delta} \leq C_{\delta}
$$


is uniformly bounded on $[0, T]$. Also,

$$
\int_{\delta}^{1-\delta} \frac{3 \sec R}{f^{2}} w d x \leq C \int_{\delta}^{1-\delta} w d x
$$

Let

$$
L_{0}=\sup _{[\delta, 1-\delta] \times[0, T]} \frac{1+\sec ^{2} R}{f^{2}}<\infty .
$$

We conclude from above that

$$
\frac{d}{d t} \int_{\delta}^{1-\delta} \log w=-\int_{\delta}^{1-\delta} w_{x}^{2} d x+L_{0} \int_{\delta}^{1-\delta} w^{2} d x+C\left(\int_{\delta}^{1-\delta} w d x+1\right)
$$

Let us choose the number $l_{0}>0$ so that the Poincaré inequality

$$
\int_{I} u_{x}^{2} d x>L_{0} \int_{I} u^{2} d x, \quad L_{0}=\left(\frac{\pi}{l_{0}}\right)^{2}
$$

holds on any interval $I$ of length $|I|<l_{0}$ and for any smooth function $u$ which is zero on the boundary of $I$. Assuming that $\sup _{t \in[0, T)} w_{l_{0}}^{*}(t)<\infty$ for this chosen number $l_{0}$ and choosing $\theta>\sup _{t \in[0, T)} w_{l_{0}}^{*}(t)$, the definition of $w_{l_{0}}^{*}$ implies that

$$
\{x \in[\delta, 1-\delta] \mid w(x, t)>\theta\}=\cup_{k} I_{k}
$$

where $I_{k}$ are disjoint open intervals of length $\left|I_{k}\right|<l_{0}$. Let $J=[\delta, 1-\delta] \backslash$ $\cup_{k} I_{k}$. Then,

$$
\begin{aligned}
\int_{\delta}^{1-\delta} w^{2} d x & =\int_{\delta}^{1-\delta}(w-\theta)^{2}+2 \theta w-\theta^{2} d x \\
& \leq \sum_{k} \int_{I_{k}}(w-\theta)^{2} d x+\int_{J}(w-\theta)^{2} d x+\int_{\delta}^{1-\delta} 2 \theta w d x
\end{aligned}
$$

Since $w-\theta=0$ at the boundary of $I_{k}$, by the Poincaré inequality (6.17)

$$
\sum_{k} \int_{I_{k}} w_{x}^{2} d x>L_{0} \sum_{k} \int_{I_{k}}(w-\theta)^{2}
$$

Hence from (6.16) and (6.18), we have

$$
\frac{d}{d t} \int_{\delta}^{1-\delta} \log w \leq C\left(\int_{\delta}^{1-\delta} w d x+1\right)
$$


implying that

$$
\int_{\delta}^{1-\delta} \log w(x, t) d x \leq C\left(\int_{0}^{T} \int_{\delta}^{1-\delta} w d x+T\right)+\int_{\delta}^{1-\delta} \log w(x, 0) d x
$$

for all $t \in[0, T)$. The proof of (6.11) now readily follows from (6.13) and the observation that $\int_{\delta}^{1-\delta} \log w(x, 0) d x<\infty$.

Lemma 6.4. Under the assumptions of Theorem 6.1, if $\sup _{t \in[0, T)} w_{l_{0}}^{*}(t)<$ $\infty$, then

$$
\sup _{t \in[0, T)} \int_{0}^{1} w(x, t)^{2} d x<\infty
$$

Proof. Let $\delta>0$ be as in (6.7). It is sufficient bound $\int_{\delta}^{1-\delta} w^{2} d x$. By direct computation, using (6.4), we have

$$
\begin{aligned}
\frac{d}{d t} \int_{\delta}^{1-\delta} w^{2} d x= & \int_{\delta}^{1-\delta} 2 w w_{t} d x=\int_{\delta}^{1-\delta} 2 w^{3} w_{x x} d x-\int_{\delta}^{1-\delta} \frac{4 w^{3} w_{x} \tan R}{f} d x \\
& +\int_{\delta}^{1-\delta} \frac{2 w^{4} \sec ^{2} R}{f^{2}}+\frac{4 w^{3} \sec R}{f^{2}}+\frac{2 w^{2}}{f^{2}} d x
\end{aligned}
$$

Integrating by parts, using that

$$
\left(\frac{\tan R}{f}\right)_{x}=\frac{1}{f^{2}}+\frac{\sec R}{w f^{2}}
$$

we obtain

$$
\begin{aligned}
\frac{d}{d t} \int_{\delta}^{1-\delta} w^{2} d x= & -6 \int_{\delta}^{1-\delta} w^{2} w_{x}^{2} d x+\int_{\delta}^{1-\delta} \frac{w^{4}\left(1+2 \sec ^{2} R\right)}{f^{2}} d x \\
& +\int_{\delta}^{1-\delta} \frac{5 w^{3} \sec R}{f^{2}} d x+\int_{\delta}^{1-\delta} \frac{2 w^{2}}{f^{2}} d x \\
& +\left.2 w^{3} w_{x}\right|_{\delta} ^{1-\delta}-\left.\frac{\tan R}{f} w^{4}\right|_{\delta} ^{1-\delta}
\end{aligned}
$$

Similarly to the proof of Lemma 6.4,

$$
2 w^{3} w_{x}\left|{ }_{\delta}^{1-\delta}-\frac{\tan R}{f} w^{4}\right|_{\delta}^{1-\delta} \leq C
$$


is uniformly bounded on $[0, T]$. Also, let $C>0$ sufficiently large so that

$$
\frac{1+2 \sec ^{2} R}{f^{2}} \leq C, \quad \frac{5 \sec R}{f^{2}} \leq C, \quad \frac{2}{f^{2}} \leq C, \quad \text { on }[\delta, 1-\delta] \times[0, T) .
$$

Since $w^{3} \leq w^{4}+1$ and $w^{2} \leq w^{4}+1$, we conclude the estimate

$$
\frac{d}{d t} \int_{\delta}^{1-\delta} w^{2} d x \leq-4 \int_{\delta}^{1-\delta} w^{2} w_{x}^{2} d x+C_{1} \int_{\delta}^{1-\delta} w^{4} d x+C
$$

with $C<\infty$ and $C_{1}<\infty$ constants independent of $t$.

Next, fix $t \in(0, T)$. For a number $\theta>1$ to be chosen momentarily, we define $J=\{x: w(x, t)>\theta\}$ so that by Lemma 6.3, we have

$$
|J| \log \theta \leq \int_{J} \log w \leq M:=\sup _{t \in[0, T)} \int_{0}^{1}[\log w]^{+}(x, t) d x .
$$

The Poincaré inequality implies that

$$
\int_{J} w^{2} w_{x}^{2} d x \geq\left(\frac{2 \pi}{|J|}\right)^{2} \int_{J}\left(w^{2}-\theta^{2}\right)^{2} d x .
$$

Hence, by choosing $\theta=\exp \left(\frac{\sqrt{C_{1}} M}{4 \pi}\right)$, we obtain

$$
-4 \int_{J} w^{2} w_{x}^{2} d x+C_{1} \int_{J}\left(w^{2}-\theta^{2}\right)^{2} d x \leq 0,
$$

which combined with (6.20), after expanding the square, gives

$$
\frac{d}{d t} \int_{\delta}^{1-\delta} w^{2} d x+2 \int_{\delta}^{1-\delta} w^{2} w_{x}^{2} d x \leq C_{2} \int_{\delta}^{1-\delta} w^{2} d x+C
$$

for constants $C_{2}<\infty$ and $C<\infty$, depending on $\theta$, but independent of $t$. This, in particular, implies the inequality

$$
\frac{d}{d t} \int_{\delta}^{1-\delta} w^{2} d x+2 \int_{\delta}^{1-\delta} w^{2} w_{x}^{2} d x \leq C_{2} \int_{\delta}^{1-\delta} w^{2} d x+C
$$

providing the desired bound on $\int_{\delta}^{1-\delta} w^{2}(x, t) d x$, thus finishing the proof of the lemma. 
Lemma 6.5. Under the same conditions as in Lemma 6.4

$$
\int_{0}^{T} \int_{0}^{1} w^{2} w_{x}^{2} d x d t<\infty
$$

Proof. Let $\delta>0$ be as in (6.7). Then, $\left|w_{x}\right| \leq C$ is uniformly bounded on $(I \backslash[\delta, 1-\delta]) \times[0, T]$. To bound $\int_{0}^{T} \int_{\delta}^{1-\delta} w^{2} w_{x}^{2} d x d t$, we integrate (6.21) and use Lemma 6.14.

Lemma 6.6. Under the assumptions of Theorem 6.1, if $\sup _{t \in[0, T)} w_{l_{0}}^{*}(t)<$ $\infty$, then

$$
\sup _{t \in[0, T)} \int_{0}^{1} w_{x}^{2}(x, t) d x<\infty
$$

Proof. By direct computation, using (6.4), we have

$$
\begin{aligned}
\frac{d}{d t} \int_{0}^{1} \frac{w_{x}^{2}}{2} d x= & \int_{0}^{1} w_{x} w_{x t} d x=-\int_{0}^{1} w_{x x} w_{t} d x=-\int_{0}^{1} w^{2} w_{x x} \\
& \times\left(w_{x x}-\frac{2 w_{x} \tan R}{f}+\frac{w \sec ^{2} R}{f^{2}}+\frac{2 \sec R}{f^{2}}+\frac{1}{w f^{2}}\right) d x
\end{aligned}
$$

Note that in the integration by parts, we used that $w_{t}\left(x_{i}, t\right)=0$ at $x_{i}=0,1$, since $w\left(x_{i}, t\right)=0$ at $x_{i}=0,1$ for all $t$. Completing the square and dropping negative terms, we obtain the estimate

$$
\frac{d}{d t} \int_{0}^{1} \frac{w_{x}^{2}}{2} d x \leq-\int_{0}^{1} w^{2}\left(w_{x x}-\frac{w_{x} \tan R}{f}\right)^{2} d x+\int_{0}^{1} \frac{w^{2} \tan ^{2} R}{f^{2}} d x .
$$

Hence,

$$
\sup _{t \in[0, T)} \int_{0}^{1} w_{x}^{2}(x, t) d x \leq \int_{0}^{1} w_{x}^{2}(x, 0) d x+2 \int_{0}^{T} \int_{0}^{1} \frac{w^{2} \tan ^{2} R}{f^{2}} w_{x}^{2} d x d t .
$$

Let $\delta>0$ be as in (6.7). Then, $\left|w_{x}\right| \leq C$ is uniformly bounded on $(I \backslash[\delta, 1-$ $\delta]) \times[0, T]$, as we noted in the proof of Lemma 6.5. Also, the reader may verify that although $\tan R=f_{x}$ is unbounded near the boundary points of $[0,1]$, the growth estimates defining the space $C_{s}^{2+\alpha, p}\left(Q_{T}\right)$ imply that $w^{2} \tan ^{2} R \leq$ $C$ is uniformly bounded on $(I \backslash[\delta, 1-\delta]) \times[0, T]$. Since also $f \geq c>0$ on 
$Q_{T}$ and $|\tan R| \leq C$ on $[\delta, 1-\delta] \times[0, T]$, it follows from Lemma 6.5 that

$$
\int_{0}^{T} \int_{0}^{1} \frac{w^{2} \tan ^{2} R}{f^{2}} w_{x}^{2} d x d t<\infty
$$

finishing the proof of this lemma.

Proof of Theorem 6.1. For any $y \in(0,1)$ and $t \in(0, T)$, we have

$$
w(y, t)=\int_{0}^{y} w_{x}(x, t) d x \leq \int_{0}^{1} w_{x}^{2}(x, t) d x
$$

since $w(0, t)=0$. It follows that

$$
\sup _{Q_{T}} w \leq \sup _{t \in[0, T)} \int_{0}^{1} w_{x}^{2}(x, t) d x<\infty
$$

by Lemma 6.6.

\section{Acknowledgment}

We are grateful to the referee of this paper for useful suggestions and comments. P. D. was Partially supported by NSF grants 0401126 and 0354639, the Guggenheim Foundation and the EPSRC at Imperial College, London. R. H. was partially supported by NSF grant 0354639 .

\section{References}

[1] B. Andrews, Contraction of convex hypersurfaces in Euclidean space, Calc. Var. Partial Diff. Equations 2 (1994), no. 2, 151-171.

[2] B. Andrews, Pinching estimates and motion of hypersurfaces by curvature functions, Preprint.

[3] S. Diater, Nonlinear degenerate curvature flows for weakly convex hypersurfaces, Calc. Var. Partial Differential Equations 22 (2005), no. 2, 229-251.

[4] M. C. Caputo and P. Daskalopoulos, The highly degenerate harmonic mean curvature flow, Preprint.

[5] P. Daskalopoulos and R. Hamilton, The free boundary on the Gauss curvature flow with flat sides, J. Reine Angenw. Math. (1999) no. 510, $187-227$. 
[6] N. V. Krylov and N. V. Safonov, Certain properties of solutions of parabolic equations with measurable coefficients. Izvestia Akad. Nauk. SSSR 40 (1980), 161-175.

[7] G. M. Liberman, Second Order Parabolic Differential Equations, World Scientific, 1996.

[8] M. Gage and R. S. Hamilton, The heat equation shrinking convex plane curves. J. Diff. Geom. 23 (1986), no. 1, 69-96.

[9] A. Friedman and B. McLeod, Blow-up of solutions of nonlinear degenerate parabolic equations, Arch. Rat. Mech. Anal. 96 (1986), $55-80$.

[10] M. E. Gage, On the size of the blow-up set for a quasilinear parabolic equation, in geometry and Nonlinear Partial Differential Equations' (Fayetteville, AR, 1990), Contemp. Math. 127, Amer. Math. Soc., Providence, RI, 1992, 41-58.

Department of Mathematics

Columbia University

NEW YORK

USA

E-mail address: pdaskalo@math.columbia.edu

Department of Mathematics

Columbia University

NEW YORK

USA

E-mail address: hamilton@math.columbia.edu

Received November 8, 2005 
